ARTICLE

\title{
Somatic PMK-1/p38 signaling links environmental stress to germ cell apoptosis and heritable euploidy
}

Najmeh Soltanmohammadi, ${ }^{1,2,3}$, Siyao Wang ${ }^{1,2,3}$ \& Björn Schumacher (iD ${ }^{1,2 凶}$

Inheritance of stable and euploid genomes is a prerequisite for species maintenance. The DNA damage response in germ cells controls the integrity of heritable genomes. Whether and how somatic stress responses impact the quality control of germline genomes has remained unclear. Here, we show that PMK-1/p38-mediated stress signaling in intestinal cells is required for germ cell apoptosis amid ionizing radiation (IR)-induced or meiotic DNA double strand breaks (DSBs) in C. elegans. We demonstrate that intestinal PMK-1/ p38 signaling regulates the germ cell death in response to environmental stress. The PMK-1/ p38 target SYSM-1 is secreted from the intestine into the germline to trigger apoptosis of meiotic pachytene cells. Compromised PMK-1/p38 signaling in intestinal cells leads to stressinduced aneuploidy in the consequent generation. Our data suggest that somatic stress surveillance controls heritable genome integrity and euploidy.

\footnotetext{
${ }^{1}$ Institute for Genome Stability in Ageing and Disease, Medical Faculty, University Hospital and University of Cologne, Joseph-Stelzmann-Str. 26,50931 Cologne, Germany. ${ }^{2}$ Cologne Excellence Cluster for Cellular Stress Responses in Ageing-Associated Diseases (CECAD), Center for Molecular Medicine Cologne (CMMC), University of Cologne, Joseph-Stelzmann-Str. 26, 50931 Cologne, Germany. ${ }^{3}$ These authors contributed equally: Najmeh

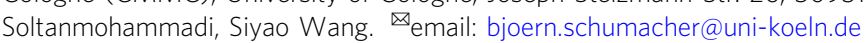


T he maintenance of genomic integrity in germ cells is a prerequisite for fertility and species maintenance. Cells invoke the DNA damage responses (DDR) to induce cell cycle arrest to allow time for the DNA repair machineries to remove the damage or to eliminate genomically compromised cells through apoptosis ${ }^{1}$. In the germ cells, integration of efficient meiotic checkpoint signaling with the canonical DDR ensures the maintenance of numerical chromosomal composition, which is crucial for successful reproduction and prevention of birth defects $^{2}$. In Caenorhabditis elegans, the evolutionary highly conserved DDR functions in the germline, which is the only proliferative tissue in adult worms ${ }^{3}$. Upon ionizing radiation (IR), the DDR induces cell cycle arrest in mitotic cells in the distal germ stem cell compartment, while late meiotic pachytene cells carrying DSBs succumb to apoptosis ${ }^{3}$. In contrast to germ cells, somatic tissues are highly radioresistant ${ }^{3-5}$. In the germline, checkpoint signaling is initiated when the conserved 9-1-1 complex proteins including MRT-2 (RAD1), HPR-9 (RAD9), and HUS-1 (HUS1) recognize DNA double-strand breaks (DSBs) ${ }^{6,7}$. DNA damage-induced germ cell apoptosis is triggered by the activation of the C. elegans p53 homolog, CEP-1, which transcriptionally induces the expression of the $\mathrm{BH}-3$ only domain genes egl-1 and ced-13,8-10. EGL-1 and CED-13 then bind to the Bcl-2 homolog CED-9 consequently unleashing the Apaf1-like CED-4 to activate the apoptosis executioner caspase CED-36,10,11.

Similar to IR-induced DNA damage, germ cell apoptosis is triggered when unresolved DSBs result from meiotic recombination failure that occurs when homologous chromosomes are unpaired and/or unsynapsed resulting in incomplete crossovers $(\mathrm{COs})^{12}$. This apoptotic response is evident in mutants, such as syp-2, that are incapable of forming proper synaptonemal complexes (SCs). In such mutants, homologous chromosomes fail to align tightly during the pachytene phase of meiosis resulting in unresolved, persistent meiotic DSBs ${ }^{13-16}$.

In C. elegans as well as in mammals, mitogen-activated protein kinase (MAPK) signaling pathways play a critical role in the regulation of apoptosis ${ }^{17-20}$. It has been suggested that, while ERK1/2 MAPK predominantly facilitates cell survival, the JNK and p38 MAPKs, both characterized as stress-responsive kinases, promote apoptosis. Recent studies, however, have challenged this simplistic view and suggested a more context and cell typespecific impact of the various MAPK pathways in the regulation of apoptosis in mammals ${ }^{21}$. It was previously reported that the ERK MAPK homolog, MPK-1, is required in C. elegans to promote germ cell apoptosis in response to DNA damage ${ }^{18}$. Moreover, we have shown that MPK-1 induces the expression of immune genes and regulates germline DNA damage-induced systemic stress resistance (GDISR) ${ }^{22}$. Both findings suggest that the role of MAPKs in modulating DDRs is evolutionary conserved. The p38 homolog PMK-1 is the terminal MAPK of a stress and also immune responsive signaling pathway whose activity seems to be restricted to somatic tissues, particularly the intestine as well as neurons ${ }^{23-26}$. PMK-1 was shown to regulate the innate immune response to pathogen infection ${ }^{27}$ and is required to induce somatic stress resistance in response to intestinal infection by Pseudomonas aeruginosa ${ }^{22}$. In addition, the induction of germline apoptosis in response to Salmonella enterica infection is dependent on PMK-1 ${ }^{20}$. While PMK-1 was demonstrated to be required in response to DNA damage for induction of germ cell apoptosis ${ }^{28}$, it has remained unclear how PMK-1/p38 impacts the regulation of germ cell apoptosis.

Here, we report that the PMK-1/p38 MAPK stress-responsive pathway in intestinal cells is required to induce germ cell apoptosis in response to IR- and persistent meiotic DNA damage in $C$. elegans. We determine that PMK-1/p38 acts upstream of CED-9/ $\mathrm{Bcl}-2$ for the regulation of DNA damage-induced germ cell apoptosis. Intriguingly, intestinal $\mathrm{PMK}-1 / \mathrm{p} 38$ regulates the synergistic effect of heat stress in elevating DNA damage-induced germ cell apoptosis, suggesting an intestinal stress surveillance mechanism that regulates the DDR in the germline. We demonstrate that the PMK-1 target SYSM-1 is secreted from the intestine and mediates the non-cell-autonomous control of DNA damage-induced germ cell apoptosis. Finally, intestinal PMK-1/ p38 was crucial to ensure the accurate inheritance of homologous $\mathrm{X}$ chromosomes upon heat stress or DNA damage. We propose that PMK-1/p38-mediated somatic stress surveillance regulates meiotic chromosomal quality control thus promoting heritable diploidy. Taken together, our data establish that somatic stress signaling regulates chromosome inheritance.

\section{Results}

PMK-1 regulates germline apoptosis in response to DNA damage. To characterize the role of $\mathrm{PMK}-1 / \mathrm{p} 38$ stress signaling in DNA damage-induced germ cell apoptosis in C. elegans, we used the mutant pmk-1(km25) and exposed it to 90 Gy of IR. To detect apoptotic corpses, worms were immobilized and germlines were visualized under the Nomarski, differential interference contrast (DIC) microscopy. Apoptotic corpses in the C. elegans germline loop can be monitored during the late pachytene stage of meiosis prophase I and can be distinguished from normal germ cells through a distinct morphological change into a disc-shaped format and an increased refractivity ${ }^{3,29,30}$. In line with previous reports, the germ cell death upon IR-induced DNA damage was blunted in pmk-1(km25) but not in jnk-1(gk7) mutant worms (Fig. 1A, B $)^{28}$. Next, we hypothesized that PMK-1 could regulate germ cell apoptosis through its known downstream transcription factor ATF-7, an ortholog of mammalian ATF2/ATF7 ${ }^{31}$. Indeed, atf-7(qd22,qd130) loss-of-function mutant worms recapitulated the apoptotic defect of $p m k-1(\mathrm{~km} 25)$ mutants in response to DNA damage (Fig. 1B). Therefore, we conclude that PMK-1/p38 MAPK signaling is required to induce germ cell apoptosis in response to DNA damage through its downstream transcription factor ATF-7.

Taken into account that PMK-1 expression appears to be restricted to somatic tissues including neurons and the intestine and that intestinal PMK-1 particularly regulates innate immunity against pathogen infection ${ }^{25,26}$, we wondered whether PMK-1mediated signals from the intestine to the germline might impact germ cell apoptosis in response to DNA damage through a noncell-autonomous mechanism. In order to test this possibility, we first verified the expression pattern of PMK-1 by using an antibody specifically against an evolutionary conserved PMK/p38 phosphorylation site ( $\mathrm{p}-\mathrm{PMK}-1)$, to detect the active form of $\mathrm{PMK}-1 / \mathrm{p} 38$. Consistent with previous reports, our immunofluorescence result confirmed that the p-PMK-1 is expressed exclusively in somatic cells, particularly the anterior end of the intestine, while this signal is completely abolished in the pmk-1 mutant (Fig. 1C). Intriguingly, upon IR-induced DNA damage, the level of p-PMK-1 is significantly enhanced (Fig. 1C), and no longer restricted to the anterior end, but instead detectable throughout the nuclei of the intestinal cells (Fig. 1C). Consistent with our immunofluorescence result, we found increased p-PMK1 levels compared to non-treated WT animals 2 to $3 \mathrm{~h}$ post-IR treatment, indicating its activation after IR-induced DNA damage (Supplementary Fig. 1A). In order to investigate the role of intestinal PMK-1, we employed a transgenic strain expressing PMK-1 under the control of the intestine-specific vha-6 promoter $^{32}$ in the pmk-1(km25) mutant background (Supplementary Fig. 1B). In line with our hypothesis, intestine-specific PMK-1 expression in the pmk-1(km25) mutant was sufficient to restore germ cells apoptosis upon IR-induced DNA damage (Fig. 1D). 
A

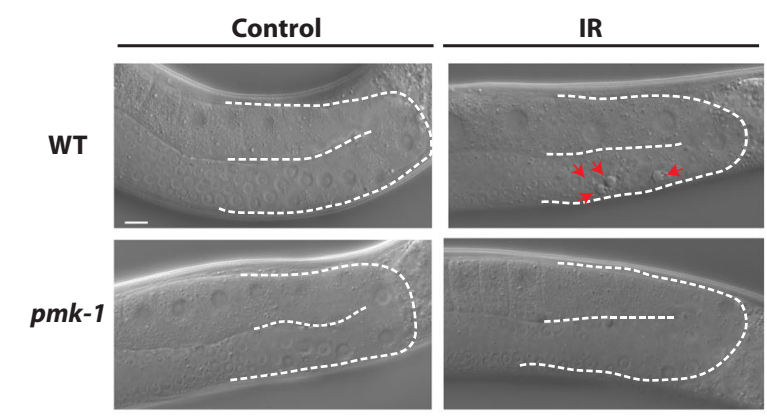

C
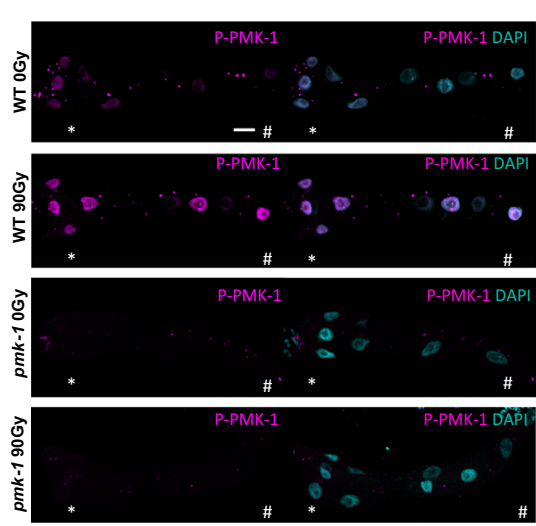

D

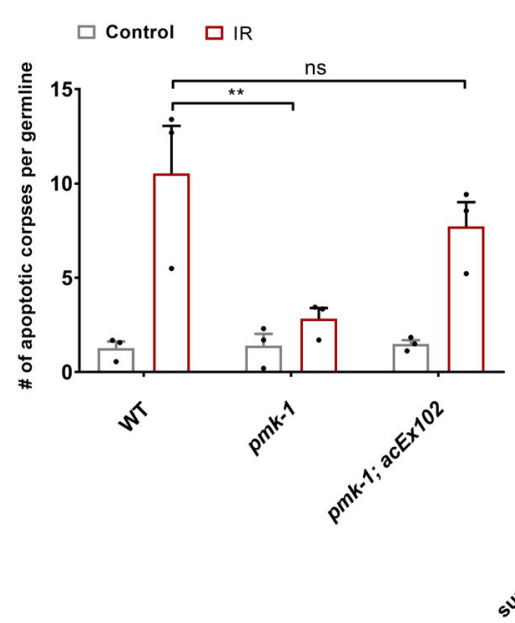

E
B

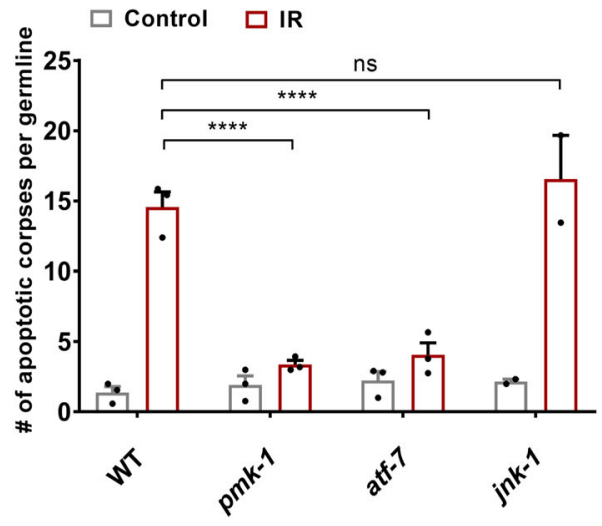

Germline

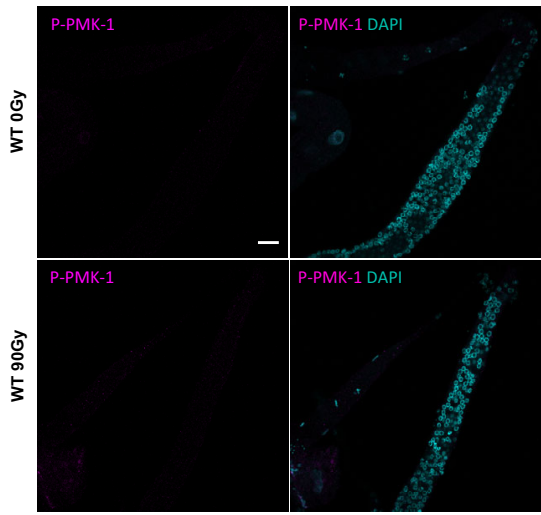

Baseline level

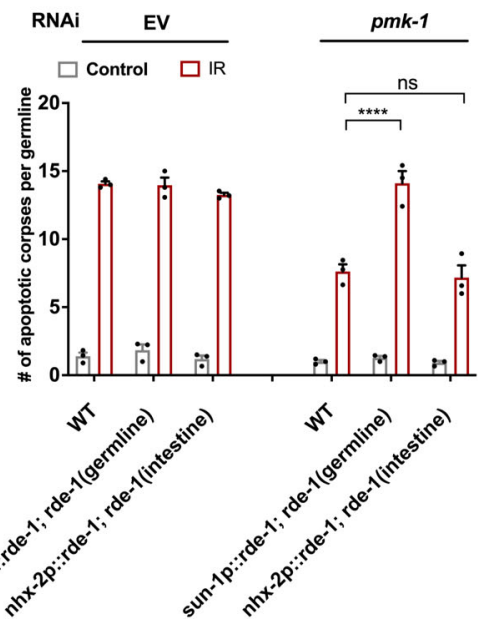

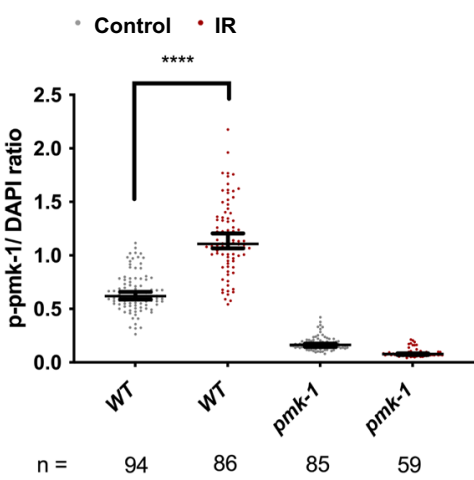

$\mathbf{F}$
To independently validate the tissue-specific role of PMK-1, we next performed a tissue-specific RNA interference (RNAi) experiment. To this end, we employed $r d e-1$ mutant strains whose RNAi deficiency is rescued by cell type-specific expression of RDE-1 expressed either exclusively in the germ cells or the intestine. The promoters of $n h x-2$ and sun- 1 were previously used to drive tissuespecific expression of $\mathrm{RDE}-1$ in the intestine and germline, respectively ${ }^{33-35}$. RNAi knockdown of pmk-1 in the intestine, but not in the germline, resulted in a similar reduction of IR-induced germ cell apoptosis as ubiquitous knockdown of pmk-1 (Fig. 1E). These data indicate that intestinal PMK-1/p38 MAPK signaling is essential to mediate DNA damage-induced germ cell apoptosis through a non-cell-autonomous signaling pathway.

Next, we investigated whether the PMK-1/p38 MAPK stress signaling pathway induces apoptosis not only upon exogenously induced DSBs but also upon endogenous meiosis-specific DSBs. To address this question, we crossed pmk-1(km25) to syp2(ok307) mutant worms that fail to properly form the 


\begin{abstract}
Fig. 1 Intestinal PMK-1/p38 regulates germ cell apoptosis in response to DNA damage through a non-cell-autonomous mechanism. A Representative DIC images of IR and untreated WT and pmk-1 $(\mathrm{km} 25)$ mutant germlines taken $6 \mathrm{~h}$ post-IR irradiation. WT worms displayed increased numbers of apoptotic corpses in the gonad loop of the germline (highlighted by red arrows). The scale bar corresponds to $20 \mu \mathrm{m}$. B Quantification of apoptotic corpses per germline revealed suppressed levels of apoptosis in pmk-1(km25) and atf-7(qd22,qd130) mutant animals, whereas the apoptosis levels in jnk-1( $\mathrm{gk} 7)$ mutant animals was comparable to those in WT worms in response to IR. (Two-way ANOVA with Tukey's multiple comparison test. WT(IR): pmk-1(IR)

${ }^{\star \star \star \star} p<0.0001, W T(I R): a t f-7(I R)^{\star \star \star \star} p<0.0001, W T(I R): j n k-1(I R)$ ns: $\left.p=0.8661\right)$. C Immunofluorescence staining of $p$-PMK-1 (violet) in dissected intestine and germline of the indicated strains with or without IR. Merged images with DAPI (turquoise) were shown. *Indicates anterior side; \#indicates posterior side. Scale bar $=20 \mu \mathrm{m}$. Right panel shows the quantification of p-PMK-1 signal intensity from C. DAPI was used for normalization. Sample sizes in C are indicated. Data are presented as median with $95 \% \mathrm{Cl}$ (Two-way ANOVA with Tukey's multiple comparisons. WT(control): WT(IR) $\left.{ }^{\star * * *} p<0.0001\right)$.

D Intestinal PMK-1 rescues the abrogated apoptosis phenotype in the pmk-1(km25) mutant animals in response to IR. (Two-way ANOVA with Tukey's multiple comparison test. WT(IR): pmk-1(IR) ${ }^{\star \star} p=0.0077, W T(I R)$ : pmk-1; acEx102(IR) ns: $p=0.5979$ ). E Tissue-specific RNAi of pmk-1 shows that intestine-specific knockdown of pmk-1 mimics the apoptotic defect of ubiquitous pmk-1 knockdown, EV treatment served as control. (Two-way ANOVA with Tukey's multiple comparison test. WT(IR): sun-1p::rde-1,rde-1(IR) $\left.{ }^{\star * \star *} p<0.0001, W T(I R): n h x-2 p:: r d e-1, r d e-1(I R), n s: p=0.9999\right)$ For B, D, E, $n=3$ independent experiments, values show mean \pm s.e.m. F pmk-1 $(\mathrm{km} 25)$ mutant animals display reduced levels of apoptosis in response to persistent recombination-mediated DNA damage inflicted by the syp-2(ok307) mutation, which can be reverted by intestine-specific expression of PMK-1 under the control of the vha- 6 promoter. $n=4$ independent experiments, values show mean \pm s.e.m. (One-way ANOVA with Tukey's multiple comparison. syp-2:syp2;pmk-1 ${ }^{\star} p=0.0365$, syp-2;pmk-1:syp-2;pmk-1;acEx102 $\left.{ }^{\star \star \star} p=0.0004\right)$. Full statistical results and exact $p$ values are shown in the Source data files.
\end{abstract}

synaptonemal complex during meiosis and lack accurate homologous chromosome alignment thus leading to persistent DSBs specifically in meiotic pachytene cells ${ }^{13}$. We observed that in contrast to syp-2(ok307) single mutant worms that were previously shown to exhibit high baseline apoptosis in germ cells, syp-2(ok307); pmk-1(km25) double mutant displayed significantly reduced levels of germ cells apoptosis (Fig. 1F). To independently verify that PMK-1 was required for the apoptotic response to endogenous DSBs in germ cells, we used also RNAi against the syp-2 and rad-51 genes. Loading of RAD-51 onto DSBs formed during meiosis promotes DNA strand invasion/ exchange, which is an essential requirement for efficient homology-directed repair ${ }^{36}$. Similarly to the absence of SYP-2, deficiency in C. elegans RAD-51 function contributes to excessive unrepaired DSBs and consequently increased level of apoptosis in germ cells ${ }^{37}$. RNAi knockdown of either rad-51 or syp-2 resulted in elevated germ cell apoptosis in wildtype (WT) but not in pmk$1(\mathrm{~km} 25)$ mutant worms (Supplementary Fig. 1C). These data indicate that PMK-1/p38 regulates germ cell apoptosis not only in response to exogenous DSBs but also in response to persistent meiotic DNA damage. Moreover, the requirement for PMK-1 for the induction of apoptosis amid DSBs that specifically occur only in meiotic pachytene cells excludes the possibility that PMK-1/ p38 might increase apoptosis as a side effect of IR damage in somatic tissues and not directly due to DSBs in the C. elegans germline. Intriguingly, expression of the acEx102 [vha-6p::pmk$1:: g f p]$ transgene in the syp-2(ok307); pmk-1(km25) double mutant background was sufficient to revert the reduced number of germ cell apoptosis observed in syp-2(ok307); pmk-1(km25) worms (Fig. 1F). Therefore, PMK-1/p38 signaling in the intestine is responsible to regulate germ cell apoptosis not only upon IRmediated DNA damage but also upon meiotic DNA damage occurring specifically in the meiotic pachytene cells.

PMK-1 regulates apoptosis in parallel or downstream of CEP1. We next examined at which level PMK-1 signaling impacts the DDR. While the apoptotic DDR in C. elegans is strictly limited to meiotic pachytene cells, DNA damage checkpoint signaling, in addition, mediates cell cycle arrest in mitotically dividing cells in the distal germline compartment. Moreover, the DNA damage checkpoint is required for DNA repair; thus, DNA damage checkpoint defects result in failure of mitotic cells to arrest, meiotic pachytene cells to undergo apoptosis, and highly elevated embryonic lethality of embryos formed by the damaged germ cells ${ }^{3,29,30}$. Comparison of F1 embryo survival of the IR-treated WT versus pmk-1(km25) and atf$7(q d 22, q d 130)$ parental generation revealed no significant differences
(Supplementary Fig. 2A). This observation suggests that PMK-1 does not impact DSB repair. Furthermore, we tested the effect of PMK-1 on cell cycle arrest regulation, which can be scored in the very distal part of the germline by counting the number of proliferating cells in the mitotic zone. As it was established previously, the number of proliferating mitotic germ cells is decreasing gradually in an IR dosage-dependent manner $3,29,30$. The same decline in the number of proliferating mitotic germ cells upon IR as in WT was also observed in the pmk-1(km25) as well as atf-7(qd22,qd130) mutant worms (Supplementary Fig. 2B). Thus, PMK-1 signaling is dispensable for the regulation of cell cycle arrest during the DDR.

We next sought to further characterize the involvement of PMK-1 signaling in the apoptotic DDR. As we have shown, IR irradiation can trigger an increase of $\mathrm{p}-\mathrm{PMK}-1$ compared to nontreated WT 2 or $3 \mathrm{~h}$ post-treatment (Supplementary Fig. 1A). However, the elevated phosphorylation of PMK-1 upon DNA damage induction was not changed in worms that were deficient for the DNA damage checkpoint protein MRT-2/RAD1 (Supplementary Fig. 1A). In summary, we conclude that the upstream components of the DNA damage checkpoint pathway are not affected by $\mathrm{PMK}-1 / \mathrm{p} 38$ MAPK activity. Instead, PMK-1/p38 is specifically involved in the apoptotic branch of the DDR.

DNA damage-induced apoptosis requires the CEP-1/p53mediated transcriptional induction of the $\mathrm{BH} 3$-only genes egl-1 and ced-136,38. To determine whether PMK-1/p38 triggers germ cell apoptosis by affecting CEP-1/p53 activity, we used quantitative reverse transcription-polymerase chain reaction (qRTPCR) to test whether the suppressed levels of apoptosis upon DNA damage in pmk-1(km25) mutants might be due to compromised transcriptional induction of the CEP-1 target genes, egl-1 and ced-13,9. As demonstrated before, the mRNA expression levels of these two $\mathrm{BH} 3$-only genes were highly upregulated in response to IR in WT worms. While in cep1(lg12501) mutants, the induction of egl-1 and ced-13 transcription was completely abrogated ${ }^{6,38}$, those $\mathrm{BH} 3$-only domain genes were normally induced upon genotoxic stress in pmk-1(km25) mutant worms (Supplementary Fig. 2C). The qRT-PCR data thus imply that even though germ cell apoptosis is abrogated in pmk$1(\mathrm{~km} 25)$ mutants in response to DNA damage, CEP-1 transcriptional activity is not modulated by PMK-1 loss. Therefore, this observation suggests that PMK-1/p38 could genetically function in parallel to or downstream of CEP-1/p53 to induce germ cell apoptosis upon IR-mediated DNA damage.

We next addressed the possibility of PMK-1/p38 to have an impact downstream of CEP-1/p53, on the core apoptotic machinery level. We employed a ced-9(n1653) loss-of-function 
mutation that abrogates the activity of the anti-apoptotic Bcl-2 homolog CED-9 and generated a double mutant with pmk$1(\mathrm{~km} 25)$. The lack of CED-9/Bcl-2 leads to elevated levels of physiological as well as DNA damage-induced apoptosis in $C$. elegans ${ }^{39,40}$. Interestingly, ced-9(n1653) loss-of-function was capable of reverting the abrogated apoptosis phenotype of pmk$1(\mathrm{~km} 25)$ with and without IR treatment to an extent similar to ced-9(n1653) single mutants (Supplementary Fig. 2D). Therefore, elevated levels of apoptosis in ced-9(n1653); pmk-1(km25) double mutants demonstrate that the PMK-1/p38 MAPK signaling pathway functions upstream of core apoptotic machinery to regulate DNA damage-induced germ cell apoptosis, possibly by blocking CED-9/Bcl-2 activity.

PMK-1 target SYSM-1 (T24B8.5) mediates germ cell apoptosis. We next wished to determine how intestinal PMK-1/p38 MAPK regulates germ cell apoptosis non-cell-autonomously. It was reported that the T24B8.5 gene, which contains an N-terminal signal sequence for extracellular secretion as well as a ShK-like toxin domain is robustly up-regulated in intestines of $C$. elegans upon pathogenic bacteria infection in a PMK-1- and ATF-7dependent manner ${ }^{31}$. Based on the functional characterization below, we propose to name the T24B8.5 gene systemic stress signaling mediator (sysm-1), which we use from here onwards (a request to wormbase to rename T24B8.5 to sysm-1 has been approved). We previously showed that germlines exhibit activation of the MPK-1 pathway, which in turn induces sysm-1 mRNA expression upon IR treatment ${ }^{22}$. sysm-1 mRNA was indeed induced in response to IR in WT but not pmk-1 mutant animals (Supplementary Fig. 3A). Consistent with SYSM-1 being a downstream effector of PMK-1 signaling, we found that sysm1(ok3236) mutants showed strongly reduced germ cell apoptosis upon IR treatment (Fig. 2A). To further test whether the putative secreted peptide encoded by the sysm-1 gene might mediate intestinal regulation of germ cell apoptosis upon genotoxic stress, we constructed transgenic strains expressing sysm-1 under its endogenous as well as cell type-specific promoters. Given that the SYSM-1::GFP signal was not detectable in transgenic lines possibly due to cleavage of GFP (likely due to processing in the secretory pathway), we performed in situ hybridization on dissected intestines and germlines to confirm the tissue-specific expression of SYSM-1 in the respective tissues (Supplementary Fig. 3B). Reexpression of the SYSM-1 protein fused to a C-terminal GFP under the control of its endogenous promoter not only reverted the apoptosis defect of sysm-1(ok3236) mutants but exacerbated the apoptotic response to IR (Fig. 2B). Expression of SYSM-1::GFP under the control of an intestine-specific promoter vha-6 restored the decreased apoptosis level of SYSM-1 deficiency (Fig. 2C). In addition, germline-specific SYSM-1::GFP, which was constitutively expressed under the mex-5 promoter ${ }^{41,42}$ was also sufficient to restore IR-induced germ cell apoptosis in the sysm-1(ok3236) mutant background (Fig. 2D). These results indicate that (i) SYSM-1 is required, (ii) intestinal SYSM-1 is sufficient, and (iii) constitutive germline expression of SYSM-1 is sufficient for DNA damage-induced germ cell apoptosis.

To verify whether SYSM-1 mediates the apoptotic signaling as downstream effector of PMK-1 in response to DNA damage, we tested whether expression of SYSM-1 in the intestine, using the vha-6 promoter, could rescue the apoptotic defect of the pmk-1 mutant. Indeed, constitutive re-expression of SYSM-1 in the intestine was sufficient to restore IR-induced apoptosis in pmk-1 mutants to WT levels (Fig. 2E). Together, these observations indicate that SYSM-1 functions downstream of PMK-1/p38 MAPK signaling pathway as a putative intestinal secreted immune peptide in response to IR-induced DNA damage to promote apoptosis in germ cells in a non-cell-autonomous fashion.

Secreted intestinal SYSM-1 acts on germline apoptosis. To further investigate whether intestinal SYSM-1 mediates the IRinduced apoptosis in the germline, we aimed to visualize the distribution pattern of SYSM-1 upon IR treatment directly. Given that a SYSM-1::GFP fusion was unattainable, we generated a series of CRISPR/Cas9 knock-in strain targeting the endogenous locus. Initially, we constructed a strain carrying a V5 tag at the C-terminal of the endogenous SYSM-1 protein (Fig. 3A). This SYSM-1::V5 strain (SYSM-1) shows the comparable level of apoptosis as WT animals post-IR treatment indicating that the fusion protein is functional (Fig. 3B). Using a V5 tag-specific antibody to detect the distribution pattern of SYSM-1, we observed a punctate pattern of SYSM-1 in the anterior intestinal cells, with strongly elevated levels upon IR treatment (Fig. 3C, D and quantifications in Supplementary Fig. 4D).

As SYSM-1 is a putative secreted peptide, we were wondering if this punctate signal of SYSM-1 co-localizes with the Golgi apparatus. Indeed, by co-staining with Golgi apparatus marker, SQV8, we observed a strong co-localization between SYSM-1 and SQV8, indicating that SYSM-1 is processed and secreted via Golgi apparatus in the anterior part of the intestine (Fig. 3C). In the germline, we also detected a clear, albeit weak, signal of the SYSM-1 peptide (Fig. 3E and Supplementary Fig. 4D). However, the punctate signal of SYSM-1 is not co-localized with SQV8 in germline cells (Supplementary Fig. 4A). Taken together, these results indicate that SYSM-1 is secreted from the intestine into the germline.

As the intestine-specific expression of SYSM-1 rescued the apoptosis defect of sysm-1 mutant, we next queried whether the intestine-expressed SYSM-1 regulates germline apoptosis via secretion. To answer this question, we generated two CRISPR/ Cas9 knock-in strains replacing the endogenous promoter with the intestinal-specific vha-6 promoter and either retained or deleted the signal sequence (ss) of SYSM-1 (Fig. 3A). We first performed immunofluorescence to verify the distribution pattern of intestine-expressed SYSM-1 and $\Delta$ ss SYSM-1. We found that the $v h a-6$ promoter-driven SYSM- 1 is distributed throughout the intestine and was also detectable in the germline (Fig. 3D, E). In stark contrast, intestine-expressed $\Delta$ ss SYSM-1 was only detectable in intestinal cells, but completely abolished in germline (Fig. 3D, E and Supplementary Fig. 4D). These results establish that SYSM-1 that is generated in the intestine is secreted into the germline through recognition of the signal sequence.

To address whether secretion of SYSM-1 is required for mediating germ cell apoptosis we quantified cell death in the SYSM-1 strains. While SYSM-1::V5 was similarly proficient as intestinal and germline expressed SYSM-1, intestinal expression of SYSM-1 lacking the secretory signal sequence significantly reduced germ cell apoptosis (Fig. 3B). Therefore, SYSM-1 is secreted from the intestine into the germline where is required to facilitate DNA damage-induced apoptosis.

To test whether the presence of SYSM-1 in the germline was sufficient to trigger apoptosis independently of secretion, we generated two CRISPR/Cas9 strains carrying SYSM-1 or $\Delta$ ss SYSM-1 under the control of germline-specific promoter, mex-5p (Fig. 3A). Unlike the SYSM-1 expressed under the control of the endogenous or intestine-specific promoters, germline promoterdriven SYSM-1 was undetectable in intestinal cells but strongly expressed in germline (Supplementary Fig. 4B, C). Germline specifically expressed SYSM-1 and $\Delta$ ss SYSM- 1 were both able to induce apoptosis upon IR treatment similarly to WT (Fig. 3B). These results establish that SYSM-1 acts in the germline to 
A

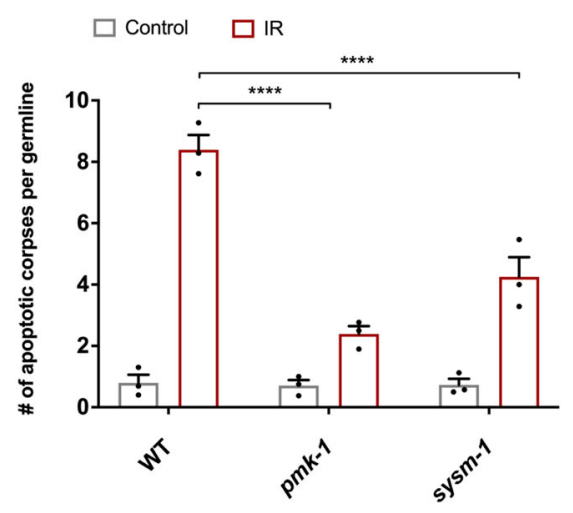

C

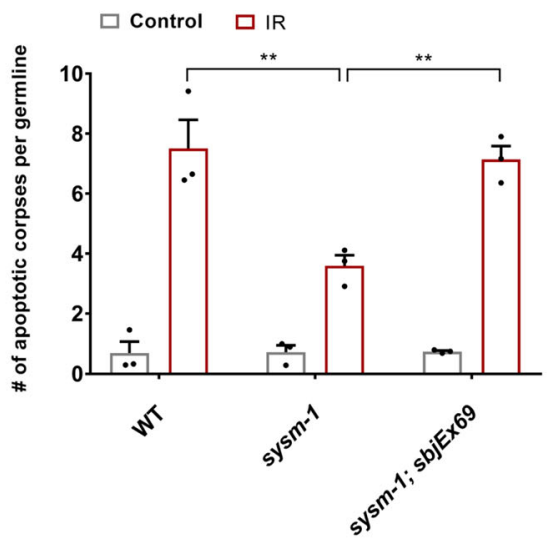

E

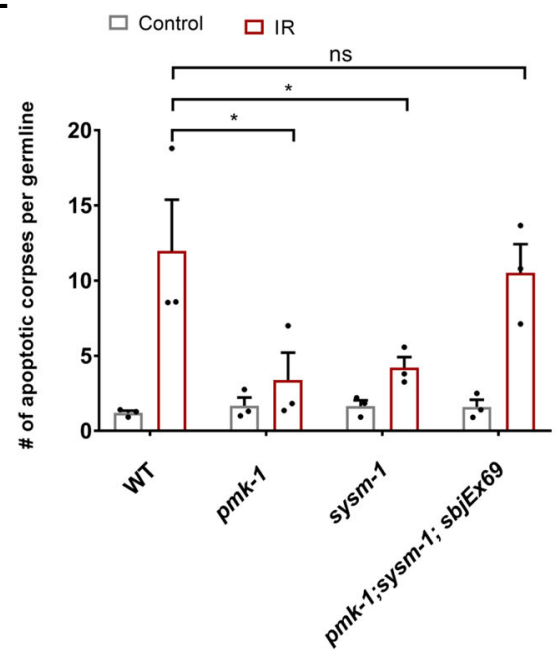

B

Endogenous (sysm-1p)

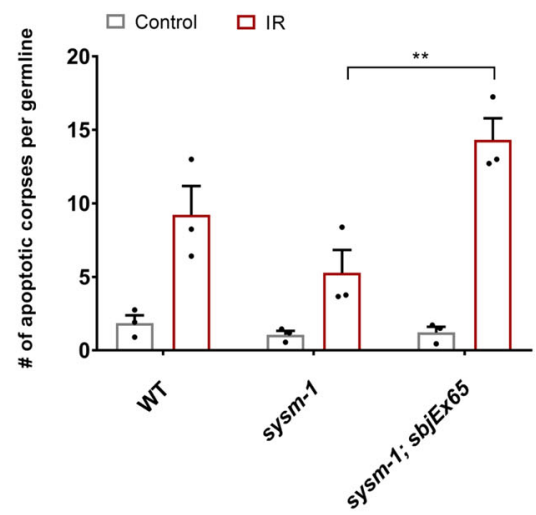

D Germline (mex-5p)

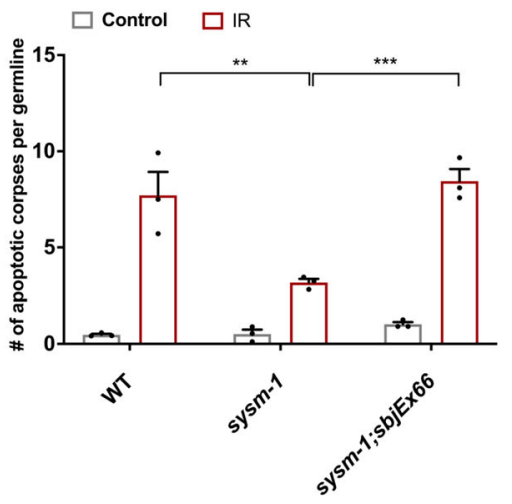

Fig. 2 PMK-1/p38 target SYSM-1 regulates germ cell apoptosis non-cell-autonomously. A sysm-1(ok3236) mutants are defective in inducing apoptosis upon IR treatment similarly to pmk-1(km25) mutants. B Endogenous-, C intestine-, and D germline-specific expression of SYSM-1 rescues the apoptosis defect in sysm-1(ok3236) mutant worms in response to IR. E Intestine-specific expression of SYSM-1 suppresses the apoptotic defect of pmk-1 mutants. The graphs A-E display $n=3$ independent experiments and each dot indicates one independent experiment that includes 8-20 germlines per condition scored $6 \mathrm{~h}$ post-IR $(90 \mathrm{~Gy})$. Error bars show mean \pm s.e.m. (Two-way ANOVA with Tukey's multiple comparison test. A $W T(I R): p m k-1(I R){ }^{\star * * *} p<0.0001$, $W T(I R): s y s m-1(I R){ }^{\star \star \star *} p<0.0001, \mathbf{B}$ sysm-1(IR):sysm-1;sbjEx65(IR) ${ }^{\star *} p=0.0022, \mathbf{C} W T(I R): s y s m-1(I R){ }^{\star *} p=0.0011$, sysm-1(IR):sysm-1;sbjEx69(IR) ${ }^{\star \star} p=0.0026, \mathbf{D} W T(I R): s y s m-1(I R){ }^{\star *} p=0.0013$, sysm-1(IR):sysm-1;sbjEx66(IR) ${ }^{\star \star *} p=0.0003, \mathbf{E} W T(I R): p m k-1(I R){ }^{*} p=0.0231, W T(I R): s y s m-1(I R)$ ${ }^{*} p=0.0465$, WT(IR):pmk-1;sysm-1;sbjEx69(IR) ns: $\left.p=0.9972\right)$. Full statistical results and exact $p$ values are shown in the Source data files. 
A

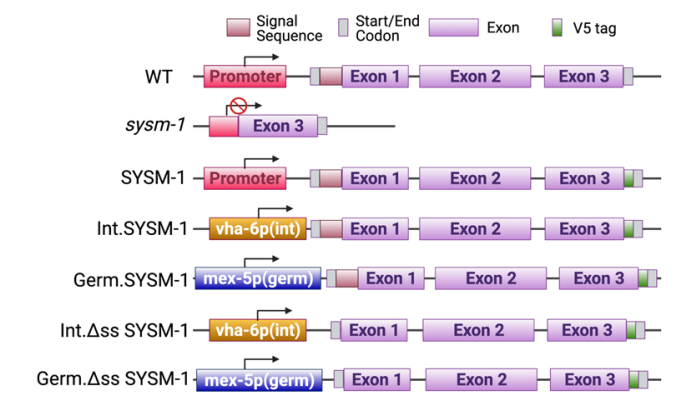

B
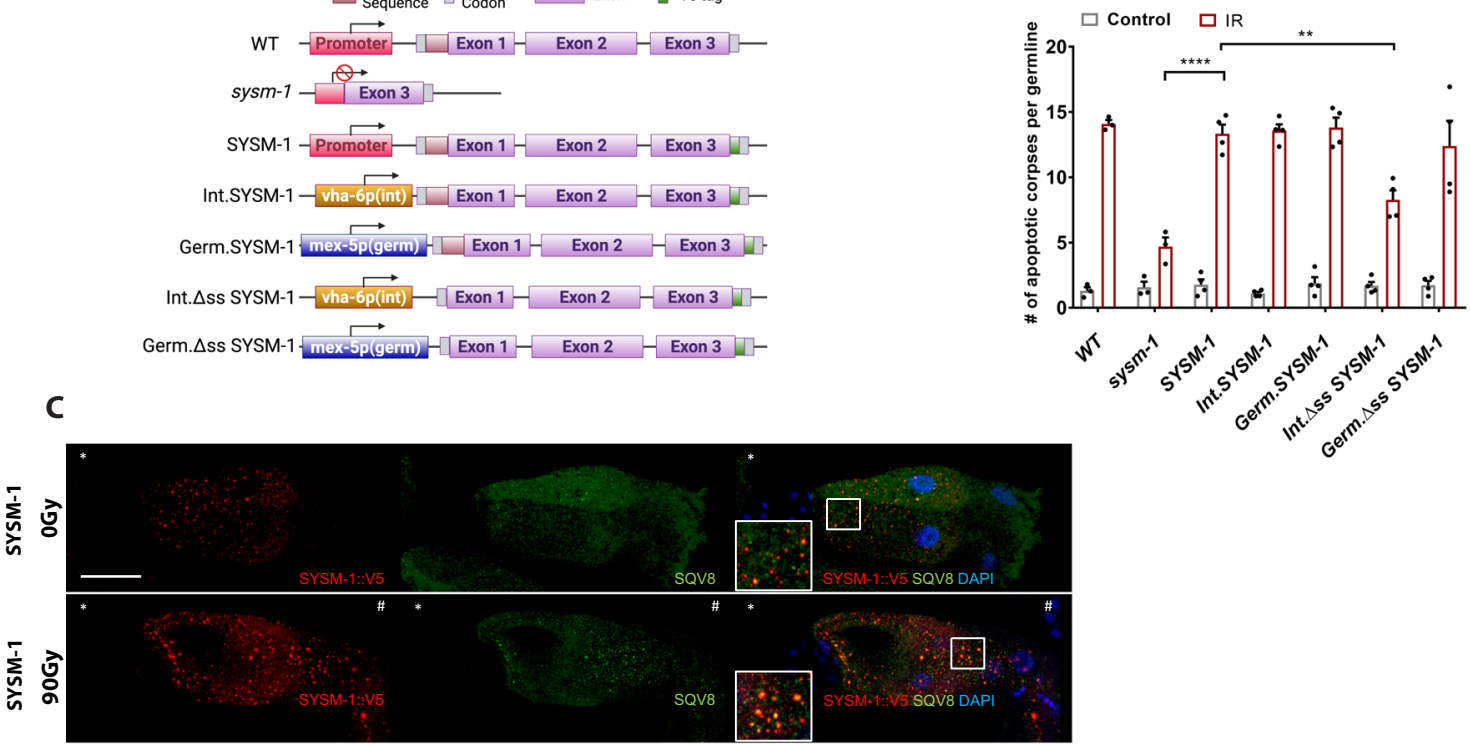

D

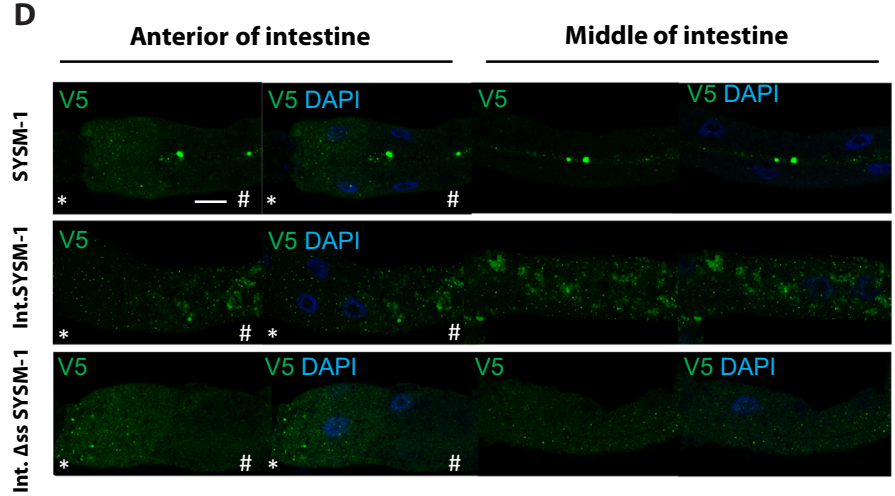

$5 \mathrm{~h}$ post IR

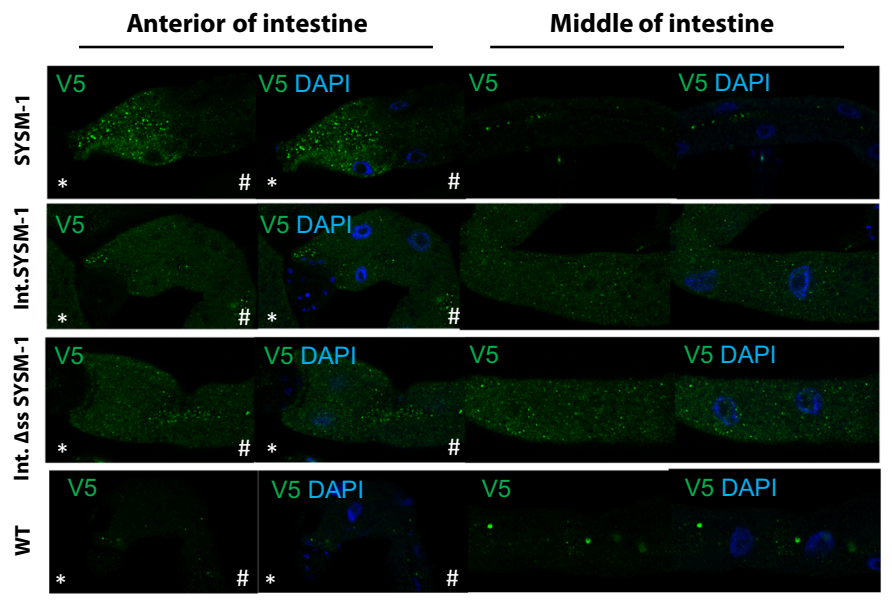

E

Non-treated

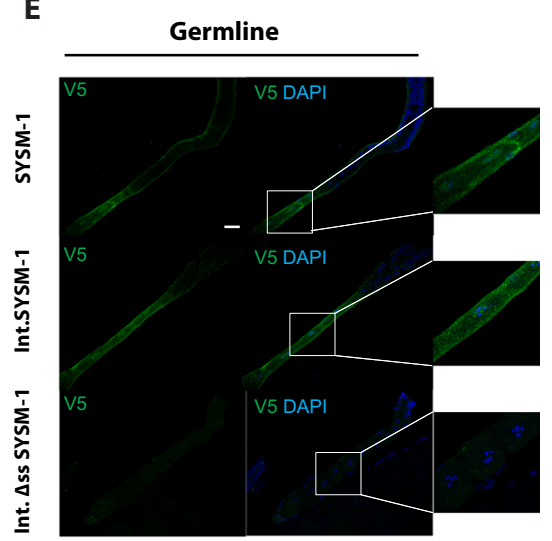

$5 \mathrm{~h}$ post IR

Germline

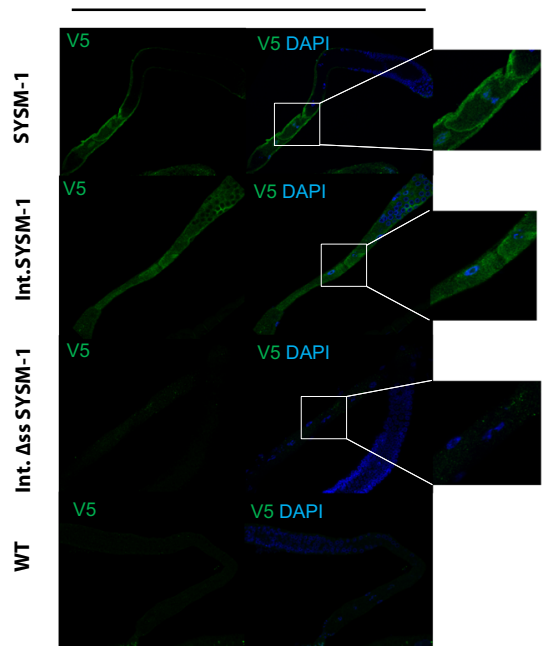

facilitate DNA damage-induced apoptosis and that the signal sequence is dispensable for the apoptotic function when SYSM-1 is expressed in the germline.

The ERK MAPK MPK-1 was previously shown to regulate DNA damage-induced apoptosis in meiotic pachytene cells ${ }^{43}$ and exert systemic effects by triggering germline DNA damage- induced systemic stress resistance (GDISR) ${ }^{22}$. We, therefore, wondered whether MPK-1/ERK signaling might interact with intestinal PMK-1/p38 signaling to regulate germ cell apoptosis. While $m p k-1$ mutants displayed elevated baseline p-PMK-1 levels in the intestine, they failed to induce intestinal p-PMK-1 further upon IR treatment (Supplementary Fig. 5A, B). We next probed 
Fig. 3 SYSM-1 is secreted from the intestine to regulate apoptosis in the germline upon IR. A Schematic representation of sysm-1 gene in WT, sysm1(ok3236) mutant and the CRISPR/Cas9-edited strains with swapping of the endogenous promoter for intestine- or germline-specific promoters and with WT or deleted signal sequence (ss). B Quantification of apoptotic corpses per germline in WT, sysm-1 and CRISPR/Cas9 strains shown in A. The graph summarizes $n=4$ independent experiments, and each dot indicates one independent experiment. Each experiment includes 10-20 germlines per condition scored $6 \mathrm{~h}$ post-IR $(90 \mathrm{~Gy})$. Error bar shows mean \pm s.e.m. $\left({ }^{\star} p<0.05,{ }^{\star \star} p<0.01,{ }^{\star \star \star} p<0.001,{ }^{\star \star \star \star} p<0.0001\right.$, Two-way ANOVA with Tukey's multiple comparison test. sysm-1(IR):SYSM-1(IR) ${ }^{\star \star \star \star} p<0.0001$, SYSM-1(IR): Int. $\Delta$ sS SYSM-1 $\left.{ }^{\star \star} p=0.001\right)$. Full statistical results and exact $p$ values are shown in the Source data files. C Representative images from immunofluorescence co-staining of SYSM-1::V5 (red) and the Golgi marker SQV8 (green). Merged images with DAPI are shown at the right side, and co-localized foci in the frame are zoomed in and shown in the corners. Scale bar $=20 \mu \mathrm{m}$. This experiment was repeated two times with similar results. D Representative images of SYSM-1::V5 staining in dissected intestines of the indicated strains with or without IR treatment are shown. ${ }^{*}$ Indicates anterior side; $\#$ indicates posterior side. Merged images with DAPI are placed at the right side. Scale bar $=20 \mu \mathrm{m}$.

E Representative images of SYSM-1::V5 staining in dissected germlines of the indicated strains with or without IR treatment are shown. Merged images with DAPI are shown at the right side. Oocyte regions in the frame are zoomed in and shown in the corners. Scale bar $=20 \mu \mathrm{m}$. WT strain was used as control, only background signal was detected. The experiments in $\mathbf{D}, \mathbf{E}$ were repeated three times with similar observations.

for the effect of hyperactivation of MPK-1/ERK. We previously showed that ALG-2 represses MPK-1/ERK activation and that alg-2 mutants show strongly exacerbated MPK-1/ERK activation in meiotic pachytene cells upon DNA damage ${ }^{44}$. However, alg-2 mutants displayed a normal IR-dependent PMK-1/p38 activation in the intestine, which was suppressed in mpk-1;alg-2 double mutants consistent with the pro-apoptotic role of MPK-1/ERK downstream of ALG-2. These data indicate a feedback loop between germline MPK-1/ERK and intestinal PMK-1/p38 where in the absence of genotoxic stress in germ cells, MPK-1/ERK limits intestinal PMK-1/p38 activation. Hyperactivation of MPK$1 /$ ERK in alg-2 mutants, however, was able to suppress the apoptotic defect of sysm-1 indicating that the MPK-1/ERKmediated activation of the apoptosome in germ cells operates downstream of PMK-1/p38-SYSM-1 signaling (Supplementary Fig. 5C). These data suggest that germline MPK-1/ERK functions downstream of the intestinal apoptotic input and reversely curbs intestinal PMK-1/p38 activation in the absence of genotoxic stress.

PMK-1 maintains heritable genomic diploidy upon heat stress. We next wondered whether suppressed DNA damage-induced apoptosis due to loss of PMK-1/p38 could affect the genomic integrity of the following generation. Therefore, we wished to clarify whether PMK-1/p38 MAPK signaling and its role in DNA damage-induced germ cell apoptosis might also be responsible for accurate meiotic chromosome segregation in response to physiological stressors. PMK-1 stress signaling has been established to regulate responses not only to pathogenic infection and oxidative stress but also upon heat stress ${ }^{24}$. Therefore, we evaluated first whether PMK-1 might regulate germ cell apoptosis in response to somatic stress conditions such as heat stress. We therefore pre-conditioned WT and pmk-1(km25) mutant worms with transient heat stress at $35^{\circ} \mathrm{C}$, before treating them with IR. This short and acute heat stress was not sufficient to increase $\mathrm{X}$ chromosome nondisjunction as male incidence was not seen in the F1 generation of WT and pmk-1(km25) mutant worms. Furthermore, the number of viable F1 progeny was not altered, while WT and pmk-1 (km25) mutant parental worms were exposed to the transient heat stress, suggesting no potential meiotic failure and aneuploidy in the next generation (Supplementary Table 1). Instead, this transient heat stress led to increased germ cell apoptosis that was further exacerbated upon IR treatment. As under heat stress, the worms have higher apoptosis levels, we limited the IR here to $30 \mathrm{~Gy}$. Therefore, we propose that the heat stress response synergizes with the DDR in the induction of germ cells apoptosis (Fig. 4A). Intriguingly, the heat stress-fueled apoptotic response was blunted in pmk$1(\mathrm{~km} 25)$ mutant animals. Specific transgenic re-expression of PMK-1 in the intestine of pmk-1(km25) mutant worms significantly restored the heat stress-induced exacerbation of germ cell apoptosis (Fig. 4A).

In addition to PMK-1, we next examined the role of SYSM-1 in response to heat stress. Similar to pmk-1(km25), sysm-1(ok3236) also shows a significant reduction of apoptosis in response to heat stress combined IR treatment, and it can be rescued by the transgenic re-expression of SYSM-1 in the intestine of sysm1(ok3236) (Fig. 4B). Moreover, intestine-specific expression of SYSM-1 is sufficient to induce heat stress-fueled apoptosis response (Fig. 4B). Taken together, we demonstrate that the intestinal PMK-1/p38 and SYSM-1 impact DNA damage-induced germ cell apoptosis upon heat stress. These results suggest that PMK-1 signaling relays somatic stress responses to impact the regulation of germ cell apoptosis through a non-cell-autonomous stress surveillance mechanism.

Next, we assessed whether the intestinal PMK-1/p38 MAPKmediated germ cell apoptosis in response to heat stress could impact euploidy of the next generation. Chromosome nondisjunction in C. elegans can result in missegregation of the $\mathrm{X}$ chromosome, which determines nematode sex as either hermaphrodites (XX) or less frequently, males (XO) ${ }^{45}$. Exposure of C. elegans hermaphrodites to $30^{\circ} \mathrm{C}$ for a time-period of $6 \mathrm{~h}$ results in the high incidence of males known as HIM phenotype in the population ${ }^{46}$. Intriguingly, we found that heat stress exposure of pmk-1 $1(\mathrm{~km} 25)$ mutant worms at $30^{\circ} \mathrm{C}$ led to a robust increase in male incidence indicating inheritance of missegregated $\mathrm{X}$ chromosomes. The intestinal PMK-1 re-expression was more than sufficient to restore the male incidence to a level comparable to WT worms (Fig. 4C). In addition to heat stress, we observed that pmk-1 (km25) mutant worms had an elevated level of male incidence in response to IR treatment which was rescued by reexpression of PMK-1 in the intestine of PMK-1-deficient worms (Fig. 4D).

In contrast to pmk-1 mutants, sysm-1(ok3236) mutants showed a strongly diminished brood size. The reduced fecundity of sysm-1 mutants was rescued by mating with WT males thus indicating a severe defect in spermatogenesis (Supplementary Fig. 6), which is further deteriorated upon heat stress or IR treatment. Only the first $\sim 10$ oocytes could be self-fertilized thus precluding the analysis of aneuploidy in the offspring following heat stress or DNA damage.

In conclusion, intestinal PMK-1/p38 MAPK signaling is required for eliminating genomically compromised germ cells to ensure heritable genomic diploidy amid environmental or genotoxic stress.

\section{Discussion}

The DNA damage checkpoint during meiosis is essential to ensure the inheritance of a stable and euploid genome. In mammals, p38 MAPK signaling regulates stress responses ranging from inflammation to DNA damage checkpoint signaling ${ }^{47}$. 
A

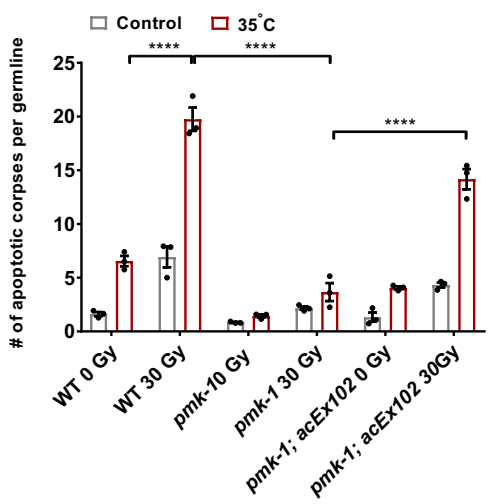

C

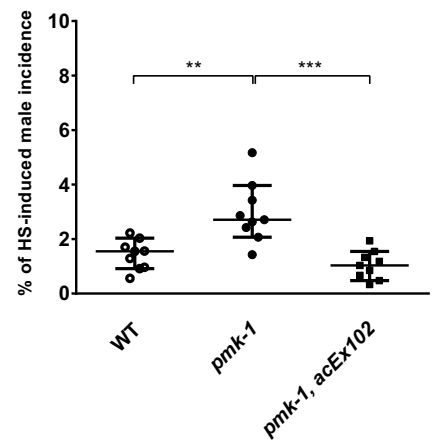

B

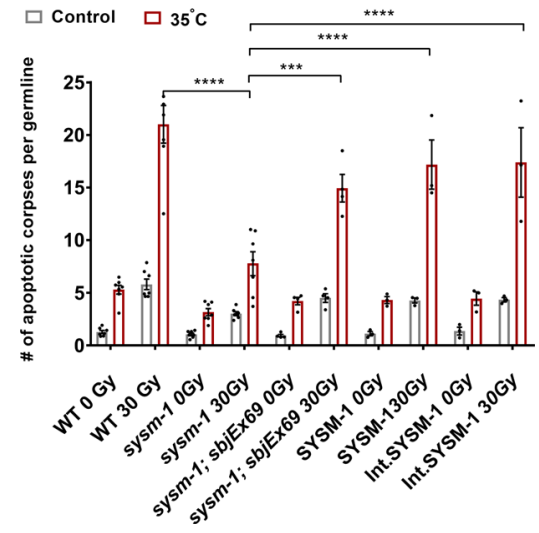

D

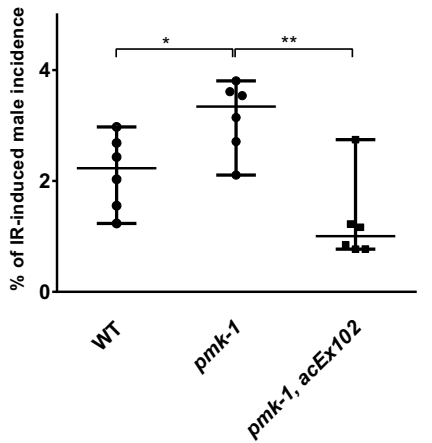

Fig. 4 Intestinal PMK-1/p38 induces germ cell apoptosis and maintains heritable euploidy upon heat stress and IR treatment. A Transient heat stress further promotes IR-induced germ cell apoptosis in an intestinal PMK-1-dependent manner. The graph summarizes $n=3$ independent experiments, and each dot indicates one independent experiment. Each experiment includes 8-15 germlines per condition scored $6 \mathrm{~h}$ post-IR (Young adult worms were heat shock treated at $35^{\circ} \mathrm{C}$ for $10 \mathrm{~min}$ and subsequently exposed to $30 \mathrm{~Gy}$ of IR). Error bars show mean \pm s.e.m. (Two-way ANOVA with Tukey's multiple

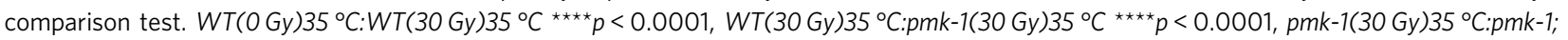

acEx102(30 Gy) $\left.35^{\circ} \mathrm{C}^{\star \star \star \star} \mathrm{p}<0.0001\right)$. B Transient heat stress further promotes IR-induced germ cell apoptosis in an intestinal SYSM-1-dependent manner. The graph summarizes $n=7$ independent experiments for the WT and sysm-1, n=4 independent experiments for sysm-1; sbjEx69, $\mathrm{n}=3$ independent experiments for SYSM-1 and Int. SYSM-1, and each dot indicates one independent experiment. Young adult worms were heat shock treated at $35^{\circ} \mathrm{C}$ for $10 \mathrm{~min}$ and subsequently exposed to $30 \mathrm{~Gy}$ of IR. Error bars show mean \pm s.e.m. (Two-way ANOVA with Tukey's multiple comparison test, WT( $30 \mathrm{~Gy}$ )

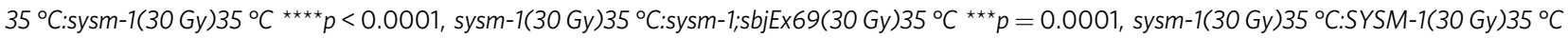

${ }^{\star * \star *} \mathrm{p}<0.0001$, sysm-1(30 Gy) $35^{\circ} \mathrm{C}$ :Int.SYSM $\left.-1(30 \mathrm{~Gy}) 35^{\circ} \mathrm{C}{ }^{\star \star * \star *} \mathrm{p}<0.0001\right)$. C Transient heat stress at $30^{\circ} \mathrm{C}$ elevates the percentage of male incidence in the F1 generation of pmk-1(km25) mutant animals, which can be rescued by intestinal-specific PMK-1 expression in pmk-1( $\mathrm{km} 25)$; acEx102 transgenic lines ( $\mathrm{L} 4$ worms heat shock treated at $30^{\circ} \mathrm{C}$ for $6 \mathrm{~h}$ and then shifted to $20^{\circ} \mathrm{C}$ ). The graph summarizes $n=9$ independent experiments each performed in biological triplicate per genotype (Sample size: $N=10$ adult worms used for $30 \mathrm{~h}$ of egg-laying experiment). (Mann-Whitney non-parametric test. pmk1:WT ${ }^{\star \star} p=0.0012$, pmk-1;acEx102:pmk-1 ${ }^{\star \star \star} p=0.0002$ ). D 90 Gy of IR treatment results in an increased percentage of male incidence in the F1 generation of pmk-1 (km25) mutant animals, which can be restored by intestine-specific PMK-1 expression in pmk-1-deficient worms (L4 worms were exposed to 90 Gy of IR). The graph summarizes $n=6$ independent experiments each performed in biological triplicate per genotype (Sample size: $\mathrm{N}=10$ adult worms used for $30 \mathrm{~h}$ of egg-laying experiment). (Mann-Whitney non-parametric test. pmk-1:WT ${ }^{\star} p=0.026, p m k-1 ; a c E x 102: p m k-1{ }^{\star *} p=0.0087$ ). Medians with $95 \% \mathrm{Cl}$ are shown in C, D. Full statistical results and exact $p$ values are shown in the Source data files.

In this study, we demonstrate that intestinal PMK-1/p38 regulates germ cell apoptosis in response to exogenous and endogenous DSBs during meiosis. Intriguingly, we found that PMK-1/p38 exacerbates DNA damage-induced germ cell apoptosis in response to heat stress suggesting that $\mathrm{PMK}-1 / \mathrm{p} 38$ in the intestine relays somatic stress surveillance to the regulation of germ cell apoptosis and thus to the control of the integrity of heritable genomes. PMK-1/p38 in C. elegans was established as stress and innate immune responsive MAPK pathway. PMK-1/p38 in intestinal cells is required for the worm's survival of Yersinia pestis infections ${ }^{27}$. In addition, the upstream PMK-1 MAPKK, SEK-1 was shown to function in the intestine and in chemosensory neurons to confer resistance to $P$. aeruginosa ${ }^{26}$. The second downstream SEK-1 MAPKK target, PMK-2 was found to modulate the behavioral response to pathogens together with PMK-1 in neurons, whereas intestinal PMK-1 alone was essential to regulate innate immune responses to pathogens ${ }^{25}$. Germ cell apoptosis in C. elegans is indeed controlled non-cellautonomously even though the mechanisms and particularly the consequences of the somatic influences have remained poorly understood. For example, neuronal HIF-1 and IRE-1 regulate CEP-1/p53, while intestinal KRI-1 functions in parallel to CEP-1/ p53, but upstream of CED-9/Bcl-2 to regulate germ cell apoptosis in response to IR-induced DNA damage ${ }^{48-50}$. We show here that PMK-1/p38 affects germ cell apoptosis upstream of the CED-9/ Bcl-2 components of the core apoptotic machinery. 
We have identified the PMK-1 target gene sysm-1 that encodes a secreted peptide and mediates the non-cell-autonomous control of germ cell apoptosis, via secretion from the intestine into the germline. We determined that endogenous p-PMK-1 and SYSM1 are both mainly expressed at the anterior end of the intestine (Figs. 1C and 3C, D). The anterior end of the intestine is anatomically located in close proximity to the bend region of germline, which is the only section of the germline where late pachytene cells undergo apoptosis. It is thus conceivable that the anterior intestinal cells secrete SYSM-1 directly into the germline to promote germ cell apoptosis in close proximity. Given that both PMK-1/p38 and MPK-1/ERK MAPKs are required for the induction of SYSM-1 in response to DNA damage it is likely that both MAPK signaling pathways interact across the germline and the soma (Supplementary Fig. 3A) ${ }^{22}$. We established previously that MPK-1/ERK exerts multiple roles in the DDR including triggering GDISR ${ }^{22}$ and facilitating apoptosis upon initial activation of the apoptosome in germ cells ${ }^{44}$. Our data suggest that in the absence of genotoxic stress, MPK-1/ERK curbs the intestinal activation of PMK-1/p38, and only upon DNA damage the two MAPK pathways are triggered and PMK-1-induced SYSM-1 mediates the MPK-1/ERK to fully activate the apoptosome in germ cells.

It is conceivable that SYSM-1 directly or indirectly impinges on CED-9/Bcl-2 activity potentially at the surface of mitochondria to block its anti-apoptotic effect in response to DNA damage. In mammals, cytokines have been reported to impinge on apoptosis by affecting the expression levels of Bcl-2 and Bcl-xL in myeloid leukemia cells ${ }^{51}$. Cytokine-mediated regulation of Bcl-2 levels and thus the balancing of apoptosis is of fundamental importance also in the maternal-fetal interaction during pregnancy. In humans, the pro-inflammatory cytokine tumor necrosis factor- $\alpha$ (TNFa) has been shown to modulate the Bcl-2 and Bax ratios thus affecting apoptosis in the endometrium and placenta. The perturbation of apoptosis by an increase of TNFa in the placenta has been linked to early pregnancy failure and premature labor in mouse, rat, and human. Importantly, various abnormal karyotypes can be observed in approximately $60 \%$ of miscarriage cases $^{52}$. It will be important to investigate whether dysregulation of apoptosis by a disturbance of the cytokine balance might be responsible for these abnormal karyotypes.

To prevent infertility, miscarriage, and birth pathologies, the maintenance of proper numerical chromosome composition across generations is of critical importance. In C. elegans, apart from the naturally occurring missegregation of $\mathrm{X}$ chromosomes in healthy XO males, trisomy of the $\mathrm{X}$ chromosome has been observed in the offspring of him mutants with various meiotic failures. Trisomy of the X chromosome results in viable worms, although with a dumpy phenotype and lower brood size and reduced fertility. Autosomal chromosome aneuploidy was first reported in the F1 generation of him- 6 mutants due to nondisjunction of chromosome IV with no obvious defect on overall physiology but reduced fertility ${ }^{45,53}$. In Drosophila melanogaster, the JNK MAPK signaling pathway inhibits the increase of aneuploidy through modulation of the pro-apoptotic gene hid, albeit only in adult cells upon IR-induced DNA damage in a p53independent manner ${ }^{54}$.

We propose that the HIM phenotype and aneuploidy of the $\mathrm{X}$ chromosome is dependent on intestinal PMK-1/p38 signaling upon heat or genotoxic stress. To our knowledge, there have never been reports of somatic stress signaling pathways affecting the heritable chromosome copy numbers in an animal species. It has been established that excessive heat exposure has negative effects on human male reproductive tissues and the spermatogenesis process and could cause infertility ${ }^{55}$. Moreover, the infertility of the Akita diabetic mice can be restored by exogenous insulin treatment. This systemic hormonal rescue of male infertility, despite incapability of passing through the blood-testis barrier, functions via the hypothalamic-pituitary-gonadal (HPG) axis to restore testosterone levels in the testis ${ }^{56}$. Recent human and animal studies have illustrated the profound negative effects of various types of prenatal stresses on gestation and physiological development of the fetus and the health span of the resulting offspring. Direct effects of stress on pregnancy can be observed via alteration in endocrine activity as well as inflammatory responses ${ }^{57}$. It was suggested that stress can modulate the hypothalamo-pituitary-adrenal (HPA) axis to elevate the production of corticotropin-releasing hormone, which in turn contributes to the induction of inflammatory cytokines during gestation $^{58}$. Maternal stress can also increase the glucocorticoid levels in utero, which can evoke stress responses in the fetus with negative effects on the development and physiology of the offspring 59 . Our study indicates an important role of somatic stress surveillance via the intestinal PMK-1/p38 signaling pathway in the regulation of meiotic chromosomal quality control and thus the inheritance of stable genomes. The Weismann barrier concept pertained that the germline is protected from somatic influences and thus genetic inheritance is only controlled autonomously by germ cells ${ }^{60}$. Our data challenge the Weismann barrier as we propose that somatic stress surveillance regulates the inheritance of chromosomes via the DDR control of the apoptotic elimination of aneuploid gametes.

\section{Methods}

C. elegans strains. All strains were maintained based on standard conditions at $20^{\circ} \mathrm{C}^{61,62}$. Strains used were N2 (Bristol; wildtype), KU25 pmk-1(km25), VC8 jnk1(gk7), ZD318 atf-7(qd22,qd130), AV276+/nT1[qIs51](IV); syp-2(ok307)/ $n T 1[q I s 51](V)$, BJS717 syp-2(ok307) V/nT1[qIs51](IV); pmk-1(km25)IV, AY102 pmk-1(km25) IV; acEx102 [vha-6p::pmk-1::GFP+rol-6(su1006)], CB5348 mrt2(e2663), XY1054 cep-1(lg12501), PP935 ced-9(n1653), BJS373 pmk-1(km25); ced9(n1653), VC2477 sysm-1 (ok3236), SD939 mpk-1(ga111) unc-79(e1068); WM53 alg-2 (ok304); BJS710 sysm-1(ok3236); sbjEX65 [sysm-1p::SYSM-1::GFP+myo2::tdTomato], BJS751 sysm-1(ok3236); sbjEx66 [mex-5p::SYSM-1::GFP+myo2::dtTomato], BJS818 sysm-1 (ok3236); sbjEx69 [vha-6p::SYSM-1::GFP+myo-2::tdTomato], BJS844 syp-2(ok307) V/nT1[qIs51](IV); pmk-1(km25)IV; acEx102 [vha6p::pmk-1::GFP+rol-6(su1006)]; BJS984 pmk-1(km25); sysm-1 (ok3236); sbjEx69 [vha-6p::SYSM-1::GFP+myo-2::tdTomato]; BJS985 sysm-1 (ok3236); alg-2 (ok304); BJS608 alg-2(ok304);mpk-1(ga111) unc-79(e1068). 5 strains were generated by SunyBiotech (China) and verified with genotyping PCR, they are: PHX3100 sysm 1(syb3100 [vha-6p::sysm-1::V5]); PHX3078 sysm-1(syb3078[mex-5p::sysm-1::V5]); PHX3073 sysm-1(syb3073[vha-6p::sysm-1(4ss)::V5]); PHX 3060 sysm-1(syb3060[mex-5p::sysm-1(Dss)::V5]); PHX2848 sysm-1(syb2848[sysm-1::V5]).

Quantification of meiotic germ cell apoptotic corpses. To quantify the number of apoptotic corpses in the meiotic late pachytene germ cells in response to DNA damage, the synchronized L4 worms were separated from the rest of the population and $20 \mathrm{~h}$ later at the young adult stage were either remained untreated or exposed to 90 Gy of IR inflicted by a cesium 137 irradiation source (Biobeam GM 8000, Eckert \& Ziegler, Gamma-Service Medical GmbH) ${ }^{3}$. $6 \mathrm{~h}$ post-IR exposure, worms were immobilized using $5 \mathrm{mM}$ levamisole (AppliChem \#A431005) and mounted on a $2 \%$ agarose pad on a microscope slide. The number of apoptotic corpses was scored via Nomarski DIC microscopy on a Zeiss Axio Imager M1/2 based on the refractive morphological changes occurring in apoptotic germ cells within the gonad loop ${ }^{29,63}$. For quantification of heat stress-induced apoptosis, young adult worms on NGM plates were exposed for $10 \mathrm{~min}$ to heat shock at $35^{\circ} \mathrm{C}$ in a preheated water bath and were treated with $30 \mathrm{~Gy}$ of IR. The number of apoptotic corpses was subsequently counted $5 \mathrm{~h}$ post-IR treatment via the same procedure as mentioned above.

Assessing mitotic germ cell cycle arrest. To assess the mitotic germ cell cycle arrest response to DNA damage, synchronized L4 larva were exposed to IR doses of 0, 30, 60, and $90 \mathrm{~Gy}$. Worms were immobilized using $5 \mathrm{mM}$ levamisole and mounted on $2 \%$ agarose pads for Nomarski DIC microscopy $16 \mathrm{~h}$ post-IR treatment. A defined region of the distal germline can be scored for either mitotic proliferative cells under normal conditions or the presence of arrested enlarged germ cell nuclei in response to IR damage. This defined field corresponds to an area of $3.125 \mu \mathrm{m} \times 6.25 \mu \mathrm{m}$ in the most distal region of the germline. For precise counting of mitotic germ cells within a specific scale, a Netmicrometer was applied 
to the microscope ocular ( $d=26 \mathrm{~mm}, 12.5 \times 12.5 / 5 ; 10$, Zeiss $)$ and the germ cell nuclei in all focal planes were counted using the Zeiss Axio Imager M1/23,10,29.

Measurement of embryonic lethality. Synchronized L4 animals were separated from the rest of the worm population by transferring them to fresh OP50-seeded plates via picking and were subsequently IR-treated with 0,30 , and $60 \mathrm{~Gy}$. Sixteen hours post-IR exposure, 5 worms from each condition were transferred to three separate OP50-seeded plates to serve as three biological replicates and were allowed to lay eggs for $2 \mathrm{~h}$. The mother worms were then removed from the plates and the number of eggs was counted. The number of surviving offspring was quantified $48 \mathrm{~h}$ after the egg-laying process to examine the embryonic hatching rate and DNA damage repair efficiency 3,29

RNAi treatment. For depletion of $p m k-1, \mathrm{rad}-51$, and syp-2 gene activities, worms were fed on HT115 E. coli bacteria harboring the RNAi plasmid (L4440) which expressed dsRNA against the desired genes from the Ahringer library. The RNAi plasmids were extracted (Nucleospin-Plasmid kit, MACHEREY-NAGEL), sequenced, and then transformed into fresh HT115 E. coli competent cells. A single colony was then selected and grown in LB medium supplemented with $50 \mu \mathrm{g} / \mathrm{ml}$ ampicillin at $37^{\circ} \mathrm{C}$ to yield an overnight culture. The next day, the HT115 overnight culture was used to inoculate a fresh culture supplemented with ampicillin that was subsequently incubated at $37^{\circ} \mathrm{C}$ until reaching an OD of 0.6 . The HT115 bacteria containing the respective RNAi plasmids were then induced with $1 \mathrm{mM}$ IPTG to trigger the production of dsRNA for $3 \mathrm{~h}$ at $37^{\circ} \mathrm{C}$. The bacteria were then pelleted with high-speed centrifugation and $1 / 3$ of the supernatant was removed. The bacterial pellet was re-suspended in the residual LB supernatant and seeded on NGM plates containing $1 \mathrm{mM}$ IPTG and was incubated overnight at room temperature ${ }^{64}$. Synchronized L1 worms of the respective genotypes were transferred to these RNAi plates and were allowed to grow at $20^{\circ} \mathrm{C}$. Counting of apoptotic corpses was performed $20 \mathrm{~h}$ post L4 stage using Nomarski DIC microscopy.

Heat-stress and IR-induced male incidence. To evaluate X chromosome missegregation and to measure male incidence rate in C. elegans, synchronized worms at L4 larval stage were transferred to fresh OP50-seeded plates and exposed to $90 \mathrm{~Gy}$ of IR or heat stress in a $30^{\circ} \mathrm{C}$ incubator for $6 \mathrm{~h}^{46} .16 \mathrm{~h}$ post-heat stress, 10 worms per each genotype were transferred to three separate OP50-seeded plates to serve as three biological replicates and were allowed to lay eggs for 5 consecutive hours. Worms were subsequently transferred to fresh OP50-seeded plates and were allowed to continue egg laying for another $25 \mathrm{~h}$ before removing the mothers from the plates (The analysis was limited to the first $30 \mathrm{~h}$ of egg laying since heat-stressed mothers proceeded to lay unfertilized oocytes after this time window). The number of surviving offspring and the number of males in the population was counted $72 \mathrm{~h}$ post-egg laying. The percentage of male incidence was quantified and compared between each genotype using the data from 9 and 6 independent experiments in Fig. $3 \mathrm{~B}$ and $\mathrm{C}$ respectively.

Plasmid constructs and generation of transgenic lines. For the generation of tissue specific and ubiquitous SYSM-1 translational reporter lines, pPD95.75, a GFP containing backbone vector, was used (Fire Lab C. elegans Vector Kit, Addgene \#1494). sysm-1genomic DNA excluding its stop codon was PCR amplified using Phusion High-Fidelity DNA-Polymerase (NEW ENGLAND BioLabs \#M0530S) and wildtype N2 genomic DNA as a template. Promoter sequences were obtained from the C. elegans Promoterome Database ${ }^{65}$. To achieve ubiquitous expression of SYSM-1 protein with a C-terminal GFP-tag, the plasmid $p B S 279$ was constructed by inserting the sysm-1 genomic DNA into the $p P D 95.75$ backbone vector. The sysm-1 promoter was inserted upstream of sysm-1::gfp. To confer germline-specific expression, the $p B S 353$ vector was generated by inserting a mex-5 promoter into the sysm-1::gfp containing backbone vector. Furthermore, the sysm-1 intestinalspecific rescue plasmid $p B S 360$ was constructed by inserting the vha- 6 promoter upstream of sysm-1::gfp. sysm-1(ok3236) worms were then injected in the germlines with $2.5 \mathrm{ng} / \mu \mathrm{l}$ of $p B S 279$ or $p B S 353$ or $p B S 360$ and $25 \mathrm{ng} / \mu \mathrm{l}$ of pBS174, myo2::tdTomato as a microinjection marker, to generate extrachromosomal array of sbjEX65 [SYSM-1P::SYSM-1::GFP + myo-2::tdTomato], sbjEx66 [mex-5P::SYSM$1:: G F P+$ myo $2:: d t$ Tomato], and sbjEx69 [vha-6P::SYSM-1::GFP + myo-2::tdTomato], respectively. The following forward and reverse primers were used: $s y s m-1$ promoter: 5' aaaaccggttttggtgatacataaatgataa $3^{\prime}, 5^{\prime} \mathrm{ttttctagaaactggacattttcctc} 3^{\prime} ;$ v ha 6 promoter: $5^{\prime}$ aaaaccggtttttatgggttttggtagg $3^{\prime}, 5^{\prime} \mathrm{ttttctagaaagtatactatttattactcgatac} 3^{\prime}$; mex-5 promoter: $5^{\prime}$ aaatctagaatctgcaagaaaatacattttcg $3^{\prime}, 5^{\prime}$ tttaccggttctctgtctgaa acattcaa3'; sysm-1 gene: 5' aaaaccggtaatgcagatttctgcattg 3', 5'tttaccggtatgcgttatctca tcggatt3

Brood size characterization. 5 synchronized L4 worms for each strain was transferred to 5 separated plates and allowed egg laying for 3 days. Worms were transferred to fresh plates every day, in order to prevent starvation. Embryos were counted every day and the total number of embryos laid in three days were considered as brood size.
Western blot analysis of phospho-PMK-1. To determine the phospho-PMK-1 level alteration upon DNA damage, 250 synchronized L4 worms from each genotype were transferred to a fresh OP50- seeded plate and were IR-treated with 0 or $90 \mathrm{~Gy} 20 \mathrm{~h}$ post-L4 at young adult stage. At the desired time points after IR treatment, worms were washed at least three times with M9 buffer to remove OP50 bacteria and were lysed directly in $70 \mu \mathrm{l}$ of $2 \times$ SDS sample buffer. Samples were subsequently boiled for $5 \mathrm{~min}$ at $95^{\circ} \mathrm{C}$ before being cooled down on ice for $10 \mathrm{~min}$. Samples were sonicated two times for $20 \mathrm{~s}$ at $60 \%$ using a Bandelin Sonopuls sonicator prior to loading for electrophoresis. After completion of $10 \%$ Bis-Tris gel electrophoresis, the protein was transferred to a nitrocellulose membrane. The membrane was then blocked using $10 \%$ BSA in $1 \times$ TBS, containing $0.1 \%$ Tween 20 (TBST) for $1 \mathrm{~h}$ at room temperature. The protein membrane was probed overnigh at $4{ }^{\circ} \mathrm{C}$ with rabbit phospho-p38 MAPK, Thr180/Tyr182 primary antibody (Cell Signaling TECHNOLOGY \#9211), which was diluted in blocking buffer with a ratio of 1:1000. The membrane was next washed at least three times with $1 \times$ TBST and incubated with peroxidase-conjugated monoclonal mouse anti-rabbit secondary antibody (Jackson ImmunoResearch \#211-032-171) with a dilution of 1:10,000 in blocking buffer for $1 \mathrm{~h}$ at room temperature. The protein membrane was then washed three times with $1 \times$ TBST prior to developing. As a loading control, the protein membrane was probed with mouse alpha-tubulin monoclonal antibody (SIGMA-ALDRICH \#T6199) overnight at $4{ }^{\circ} \mathrm{C}$ with a dilution of $1: 10,000$ in $5 \%$ milk powder in $1 \times$ PBST followed by an incubation with peroxidaseconjugated goat anti-mouse secondary antibody $(1: 10,000)$ (Jackson ImmunoResearch \#115-035-174) for $1 \mathrm{~h}$ at room temperature. The membrane was finally developed using the Amersham Enhanced Chemiluminescence (ECL) Prime Western Blotting Detection Reagent (\#RPN2232).

Immunofluorescence staining. Day 1 adult worms were picked from plates, and transferred to a drop of M9 buffer onto a $0.3 \%$ poly-lysine-treated three-well slide $(3 \times 14 \mathrm{~mm}$ printed wells slides from Fisher Scientific). Germline and intestine dissection was carried out with two syringe needles, followed by fixation with $3.7 \%$ formaldehyde for $1 \mathrm{~h}$. Then a $24 \times 24 \mathrm{~mm}$ coverslip was placed onto the drop, and the slide was left in $-80^{\circ} \mathrm{C}$ freezer for $10 \mathrm{~min}$ to perform the "freeze-cracking" procedure. Then the slide was quickly transferred to $-20^{\circ} \mathrm{C}$ methanol for $<1 \mathrm{~min}$. After fixation, slides were washed 1 time with $1 \times$ PBS and 2 times with $1 \times$ PBST. In order to improve the signal quality, slides were first blocked for $20 \mathrm{~min}$ with ImageiT FX signal enhancer (Thermo Fisher/Invitrogen, Carlsbad, CA) before blocking with $1 \times$ PBST containing $10 \%$ Donkey Serum for another $20 \mathrm{~min}$. Afterward, primary antibodies diluted with $1 \times$ PBST containing 5\% Donkey Serum were applied to the slides and incubated at $4{ }^{\circ} \mathrm{C}$ overnight. After 3 times washing with $1 \times$ PBST, the slides were incubated with secondary antibodies diluted with $1 \times$ PBST at $37^{\circ} \mathrm{C}$ for $30 \mathrm{~min}$. Then slides were washed with $1 \times$ PBST 3 times and mounted with DAPI Fluoromount-G mounting medium (Southern Biotech) and sealed with nail polish. Slides were stored at $4{ }^{\circ} \mathrm{C}$ in dark before imaging.

Primary antibodies used for immunofluorescence staining are Mouse monoclonal anti-V5 tag (SV5-P-K) antibody (Abcam, ab27671, dilution 1:50), Rabbit monoclonal anti-V5 tag (SV5-P-K) (Abcam, ab206566, dilution 1:50), rabbit phospho-p38 MAPK, Thr180/Tyr182 primary antibody (Cell Signaling TECHNOLOGY \#9211, dilution 1:100) and mouse mAb SQV8 (Developmental Studies Hybridoma Bank) at $1 \mu \mathrm{g} / \mathrm{ml}$. Secondary antibodies used are AlexaFluor 488 donkey anti-mouse IgG (Thermo Fisher/Invitrogen,Cat. No.: A21202, dilution 1:500 in PBS-T;) and AlexaFluor 594 donkey anti-rabbit IgG (Cat. No.: A21207, dilution 1:500 in PBS-T; Thermo Fisher/Invitrogen).

Fluorescence images for quantification were taken Zeiss Meta 710 confocal laser scanning microscope was used. For quantification, fixed exposure time was set for different treatments and strains. For p-PMK-1 staining, $Z$-stack images were taken with Zeiss Meta 710 confocal microscope, and the p-PMK-1 signal intensity was measured with the Imaris $\times 649.1 .2$ software. All of the fluorescence intensity was normalized to DAPI signal.

qRT-PCR. For whole worm qRT-PCR, 200 synchronized L4 worms per genotype were transferred to three separate OP50-seeded plates to serve as three biological replicates. $20 \mathrm{~h}$ later and at young adult stage, worms were IR-treated with 0 and 90 Gy. Two hours after IR treatment, worms were washed with M9 buffer to remove excess of OP50 bacteria and were lysed in $1 \mathrm{ml}$ TRIzol reagent (ThermoFisher Scientific \#15596026), supplemented with $0.7 \mathrm{~mm}$ zirconia/silica beads (Roth \#11079110z). The animals were further disrupted using Precellys24 (Bertin \#P000669-PR240-A). 1-Bromo-3-chloropropane (SIGMA-ALDRICH \#B9673) was used for phase separation prior to RNA isolation using RNeasy mini kit (Qiagen \#74104). For RNA isolation from dissected germlines and intestines, synchronized day-1 adults were dissected with syringe needles in dissection buffer containing $1.1 \times$ egg salts buffer, $0.2 \%$ Tween 20 and $20 \mathrm{mM}$ sodium azide. $25-30$ germlines or intestines per condition were collected in $1 \mathrm{ml}$ TRIzol reagent containing $0.7 \mathrm{~mm}$ zirconia/silica beads. To each sample, $20 \mu \mathrm{g}$ of glycogen (Thermo Scientific) were added before tissue disruption by vortexing for $5 \mathrm{~min}$ and RNA isolation procedure. Reverse transcription into cDNA with $1 \mu \mathrm{g}$ of isolated RNA was performed using Superscript II kit (Invitrogen \#10328062). For qRT-PCR reaction, cDNA sample was mixed with SYBR Green I (SIGMA-ALDRICH \#S9430) and Platinum Taq polymerase (Invitrogen \#10966018). All qRT-PCR reactions were performed with 2 technical replicates. qRT-PCRs were normalized to act-1, tbg-1 and vha-6 
housekeeping gene expression. For Normalization of qRT-PCR with dissected germlines, syp-2 instead of vha- 6 was used as housekeeping gene. For data analysis, the second derivative maximum method was applied, and induction of target cDNA was calculated according to the equation: $\left(E_{\text {target }} \Delta \mathrm{CP}(\mathrm{cDNAref}-\mathrm{cDNAsample})\right.$

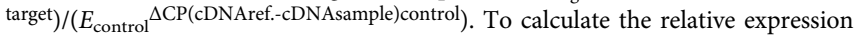
of a target gene, each sample was compared to a reference sample, which is the untreated WT. The following forward and reverse primers were used: egl-1: $5^{\prime}$ ta ctcctcgtctcaggactt $3^{\prime}, 5^{\prime}$ catcgaagtcatcgcacat $3^{\prime}$; ced-13: 5' gtcgtacaagcgtgatggat $3^{\prime}$, $5^{\prime}$ acggtgtttgagttgcaagc $3^{\prime} ;$ sysm-1: $5^{\prime}$ ctgtaacgaagcagatgttagaagtg $3^{\prime}$, 5' gggcattgttcagc aatattttcatc $3^{\prime} ;$ sysm-1 targeting ok3236 fragment: $5^{\prime}$ catcggatttgtgattgtgct $3^{\prime}, 5^{\prime} \mathrm{cgac}$ aaccacttctaacatctg $3^{\prime}$.

RNA in situ hybridization in dissected germline and intestine. The detection of sysm-1 mRNA was carried out by a modified method described by Lee and Schedl ${ }^{66}$. Briefly, full-length sysm-1 cDNA was amplified with forward primer $5^{\prime}$ atgcgttatctcatcggatttg $3^{\prime}$ and reverse primer $5^{\prime}$ catgcagatttctgcattgg $3^{\prime}$. The DIGlabeled single-strand DNA probes were generated with asymmetric (one-way) PCR, which gives anti-sense probes with reverse primer, and sense probes with forward primer.

Dissected intestines and germlines from each strain were transferred to 96-well plates with PBST. Fixation was performed with paraformaldehyde/glutaraldehyde fixation buffer for $2 \mathrm{~h}$. Tissues were then digested with Proteinase $\mathrm{K}$ for $30 \mathrm{~min}$ and washed with PBST. Tissues were pre-hybridized in hybridization buffer (5x SSC, $50 \%$ deionized formamide, $100 \mu \mathrm{g} / \mathrm{ml}$ autoclaved Herring sperm DNA, $50 \mu \mathrm{g} / \mathrm{ml}$ Heparin and $0.1 \%$ Tween-20) for $1 \mathrm{~h}$ at $57^{\circ} \mathrm{C}$. Hybridization was performed at $57^{\circ} \mathrm{C}$ for $36 \mathrm{~h}$. Then tissues were washed with the following buffers: hybridization buffer at $57^{\circ} \mathrm{C}, 50 \%$ hybridization buffer $/ 50 \%$ PBST at $57^{\circ} \mathrm{C}$, PBST at $57^{\circ} \mathrm{C}$ and PBST at room temperature. Tissues were then washed for $30 \mathrm{~min}$ in PBST prior to the detection.

Probes were detected with alkaline-phosphatase-mediated detection method. Briefly, alkaline-phosphatase-conjugated anti-DIG antibody (Roche (\#1 093 274)) was diluted in PBST/BSA buffer, and incubated with tissues overnight at $4{ }^{\circ} \mathrm{C}$. After three times washing with PBST/BSA, tissues were incubated with BCIP/NDT solution. Positive signal appeared at around $30 \mathrm{~min}-1 \mathrm{~h}$ after adding BCIP/NDT. The sense probe control gives a clean or background signal. The reaction was stopped by washing three times with PBST. Then the tissues were transferred to agar pad and mounted with the anti-fade solution. In situ hybridization images were taken with a bright field (Nomarski) microscope (ECLIPSE-Ci-E Microscope).

Data presentation and statistical analysis. All the data and statistical significances were analyzed using the GraphPad Prism 7 software package (GraphPad). Statistical methods, sample size information and error bar descriptions are reported in the figure legends. Independent repeat experiments are shown in the supplementary section. Randomization was not applied because the group allocation was guided based on the genotype of the respective mutant worms. Worms of a given genotype were nevertheless randomly selected from large strain populations for each experiment without any preconditioning. Blinding was not applied as the experiments were carried out under highly standardized and predefined conditions such that an investigator-induced bias can be excluded. Averages of independent experiments are shown with mean \pm s.e.m. When individual experiments, staining quantification, and male incidence as shown, median with $95 \% \mathrm{Cl}$ was used as these data types contain outliers, which should not be shown as mean with SD. For the rest of experiments, such as qRT-PCT data, we use the common mean \pm SD. The full statistical results are shown in Source data files.

Reporting summary. Further information on research design is available in the Nature Research Reporting Summary linked to this article.

\section{Data availability}

All the independent experimental repeats for the graphs in this paper are available within the source data file of the article. Source data are provided with this paper.

Received: 20 July 2021; Accepted: 8 January 2022;

Published online: 04 February 2022

\section{References}

1. Jackson, S. P. \& Bartek, J. The DNA-damage response in human biology and disease. Nature 461, 1071-1078 (2009).

2. MacQueen, A. J. \& Hochwagen, A. Checkpoint mechanisms: the puppet masters of meiotic prophase. Trends Cell Biol. 21, 393-400 (2011).

3. Gartner, A., Milstein, S., Ahmed, S., Hodgkin, J. \& Hengartner, M. O. A conserved checkpoint pathway mediates DNA damage-induced apoptosis and cell cycle arrest in C. elegans. Mol. Cell 5, 435-443 (2000).
4. Johnson, T. E. \& Hartman, P. S. Radiation effects on life span in Caenorhabditis elegans. J. Gerontol. 43, B137-B141 (1988).

5. Vermezovic, J., Stergiou, L., Hengartner, M. O. \& d'Adda di Fagagna, F. Differential regulation of DNA damage response activation between somatic and germline cells in Caenorhabditis elegans. Cell Death Differ. 19, 1847-1855 (2012).

6. Hofmann, E. R. et al. Caenorhabditis elegans HUS-1 is a DNA damage checkpoint protein required for genome stability and EGL-1-mediated apoptosis. Curr. Biol. 12, 1908-1918 (2002).

7. Ahmed, S. \& Hodgkin, J. MRT-2 checkpoint protein is required for germline immortality and telomere replication in C. elegans. Nature 403, 159-164 (2000).

8. Derry, W. B., Putzke, A. P. \& Rothman, J. H. Caenorhabditis elegans p53: role in apoptosis, meiosis, and stress resistance. Science 294, 591-595 (2001)

9. Schumacher, B., Hofmann, K., Boulton, S. \& Gartner, A. The C. elegans homolog of the p53 tumor suppressor is required for DNA damage-induced apoptosis. Curr. Biol. 11, 1722-1727 (2001).

10. Schumacher, B. et al. C. elegans ced-13 can promote apoptosis and is induced in response to DNA damage. Cell Death Differ. 12, 153-161 (2005).

11. Conradt, B. \& Horvitz, H. R. The C. elegans protein EGL-1 is required for programmed cell death and interacts with the Bcl-2-like protein CED-9. Cell 93, 519-529 (1998).

12. Bhalla, N. \& Dernburg, A. F. A conserved checkpoint monitors meiotic chromosome synapsis in Caenorhabditis elegans. Science 310, 1683-1686 (2005).

13. Colaiacovo, M. P. et al. Synaptonemal complex assembly in C. elegans is dispensable for loading strand-exchange proteins but critical for proper completion of recombination. Dev. Cell 5, 463-474 (2003).

14. MacQueen, A. J., Colaiacovo, M. P., McDonald, K. \& Villeneuve, A. M. Synapsis-dependent and -independent mechanisms stabilize homolog pairing during meiotic prophase in C. elegans. Genes Dev. 16, 2428-2442 (2002).

15. Smolikov, S. et al. SYP-3 restricts synaptonemal complex assembly to bridge paired chromosome axes during meiosis in Caenorhabditis elegans. Genetics 176, 2015-2025 (2007).

16. Smolikov, S., Schild-Prufert, K. \& Colaiacovo, M. P. A yeast two-hybrid screen for SYP-3 interactors identifies SYP-4, a component required for synaptonemal complex assembly and chiasma formation in Caenorhabditis elegans meiosis. PLoS Genet. 5, e1000669 (2009).

17. Bulavin, D. V. et al. Phosphorylation of human $\mathrm{p} 53$ by p 38 kinase coordinates $\mathrm{N}$-terminal phosphorylation and apoptosis in response to UV radiation. EMBO J. 18, 6845-6854 (1999)

18. Rutkowski, R. et al. Regulation of Caenorhabditis elegans p53/CEP-1dependent germ cell apoptosis by Ras/MAPK signaling. PLoS Genet. 7, e1002238 (2011).

19. Plotnikov, A., Zehorai, E., Procaccia, S. \& Seger, R. The MAPK cascades: signaling components, nuclear roles and mechanisms of nuclear translocation. Biochim. Biophys. Acta 1813, 1619-1633 (2011).

20. Aballay, A., Drenkard, E., Hilbun, L. R. \& Ausubel, F. M. Caenorhabditis elegans innate immune response triggered by Salmonella enterica requires intact LPS and is mediated by a MAPK signaling pathway. Curr. Biol. 13, 47-52 (2003).

21. Wada, T. \& Penninger, J. M. Mitogen-activated protein kinases in apoptosis regulation. Oncogene 23, 2838-2849 (2004).

22. Ermolaeva, M. A. et al. DNA damage in germ cells induces an innate immune response that triggers systemic stress resistance. Nature 501, 416-+ (2013).

23. Kim, D. H. et al. A conserved p38 MAP kinase pathway in Caenorhabditis elegans innate immunity. Science 297, 623-626 (2002).

24. Mertenskotter, A., Keshet, A., Gerke, P. \& Paul, R. J. The p38 MAPK PMK-1 shows heat-induced nuclear translocation, supports chaperone expression, and affects the heat tolerance of Caenorhabditis elegans. Cell Stress Chaperones 18, 293-306 (2013).

25. Pagano, D. J., Kingston, E. R. \& Kim, D. H. Tissue expression pattern of PMK2 p38 MAPK is established by the miR-58 family in C. elegans. PLoS Genet. 11, e1004997 (2015).

26. Shivers, R. P., Kooistra, T., Chu, S. W., Pagano, D. J. \& Kim, D. H. Tissuespecific activities of an immune signaling module regulate physiological responses to pathogenic and nutritional bacteria in C. elegans. Cell Host Microbe 6, 321-330 (2009).

27. Bolz, D. D., Tenor, J. L. \& Aballay, A. A conserved PMK-1/p38 MAPK is required in Caenorhabditis elegans tissue-specific immune response to Yersinia pestis infection. J. Biol. Chem. 285, 10832-10840 (2010).

28. Eberhard, R. et al. Ribosome synthesis and MAPK activity modulate ionizing radiation-induced germ cell apoptosis in Caenorhabditis elegans. PLoS Genet. 9, e1003943 (2013).

29. Gartner, A., MacQueen, A. J. \& Villeneuve, A. M. Methods for analyzing checkpoint responses in Caenorhabditis elegans. Methods Mol. Biol. 280, 257-274 (2004) 
30. Rieckher, M., Bujarrabal, A., Doll, M. A., Soltanmohammadi, N. \& Schumacher, B. A simple answer to complex questions: Caenorhabditis elegans as an experimental model for examining the DNA damage response and disease genes. J. Cell Physiol. 233, 2781-2790 (2018).

31. Shivers, R. P. et al. Phosphorylation of the conserved transcription factor ATF-7 by PMK-1 p38 MAPK regulates innate immunity in Caenorhabditis elegans. PLoS Genet. 6, e1000892 (2010).

32. Oka, T., Toyomura, T., Honjo, K., Wada, Y. \& Futai, M. Four subunit a isoforms of Caenorhabditis elegans vacuolar $\mathrm{H}+$-ATPase. Cell-specific expression during development. J. Biol. Chem. 276, 33079-33085 (2001).

33. Nehrke, K. A reduction in intestinal cell pHi due to loss of the Caenorhabditis elegans $\mathrm{Na}+/ \mathrm{H}+$ exchanger NHX-2 increases life span. J. Biol. Chem. 278, 44657-44666 (2003).

34. Nehrke, K. \& Melvin, J. E. The NHX family of Na+-H+ exchangers in Caenorhabditis elegans. J. Biol. Chem. 277, 29036-29044 (2002).

35. Zou, L. N. et al. Construction of a germline-specific RNAi tool in C. elegans. Sci. Rep. https://doi.org/10.1038/s41598-019-38950-8 (2019).

36. Shinohara, A., Ogawa, H. \& Ogawa, T. Rad51 protein involved in repair and recombination in S. cerevisiae is a RecA-like protein. Cell 69, 457-470 (1992).

37. Alpi, A., Pasierbek, P., Gartner, A. \& Loidl, J. Genetic and cytological characterization of the recombination protein RAD-51 in Caenorhabditis elegans. Chromosoma 112, 6-16 (2003).

38. Schumacher, B. et al. Translational repression of $C$. elegans p53 by GLD-1 regulates DNA damage-induced apoptosis. Cell 120, 357-368 (2005).

39. Xue, D. \& Horvitz, H. R. Caenorhabditis elegans CED-9 protein is a bifunctional cell-death inhibitor. Nature 390, 305-308 (1997)

40. Wu, D., Wallen, H. D. \& Nunez, G. Interaction and regulation of subcellular localization of CED-4 by CED-9. Science 275, 1126-1129 (1997).

41. Merritt, C., Rasoloson, D., Ko, D. \& Seydoux, G. 3' UTRs are the primary regulators of gene expression in the C. elegans germline. Curr. Biol. 18 1476-1482 (2008).

42. Zeiser, E., Frokjaer-Jensen, C., Jorgensen, E. \& Ahringer, J. MosSCI and gateway compatible plasmid toolkit for constitutive and inducible expression of transgenes in the C. elegans germline. PLoS ONE https://doi.org/10.1371/ journal.pone.0020082 (2011).

43. Rutkowski, R. et al. Regulation of Caenorhabditis elegans p53/CEP-1dependent germ cell apoptosis by Ras/MAPK signaling. PLoS Genet. https:// doi.org/10.1371/journal.pgen.1002238 (2011).

44. Doll, M. A., Soltanmohammadi, N. \& Schumacher, B. ALG-2/AGOdependent mir-35 family regulates DNA damage-induced apoptosis through MPK-1/ERK MAPK signaling downstream of the core apoptotic machinery in Caenorhabditis elegans. Genetics https://doi.org/10.1534/genetics.119.302458 (2019).

45. Hodgkin, J. Karyotype, ploidy, and gene dosage. WormBook 1-9 (2005).

46. Hodgkin, J. Sex determination. Right gene, wrong chromosome. Nature 336, 712 (1988).

47. Cuenda, A. \& Rousseau, S. p38 MAP-kinases pathway regulation, function and role in human diseases. Biochim. Biophys. Acta 1773, 1358-1375 (2007).

48. Ito, S., Greiss, S., Gartner, A. \& Derry, W. B. Cell-nonautonomous regulation of C. elegans germ cell death by kri-1. Curr. Biol. 20, 333-338 (2010).

49. Sendoel, A., Kohler, I., Fellmann, C., Lowe, S. W. \& Hengartner, M. O. HIF-1 antagonizes p53-mediated apoptosis through a secreted neuronal tyrosinase. Nature 465, 577-583 (2010)

50. Levi-Ferber, M. et al. It's all in your mind: determining germ cell fate by neuronal IRE-1 in C. elegans. PLoS Genet. 10, e1004747 (2014).

51. Lotem, J. \& Sachs, L. Regulation of bcl-2, bcl-XL and bax in the control of apoptosis by hematopoietic cytokines and dexamethasone. Cell Growth Differ. 6, 647-653 (1995).

52. Lea, R. G. et al. Cytokines and the regulation of apoptosis in reproductive tissues: a review. Am. J. Reprod. Immunol. 42, 100-109 (1999).

53. Hodgkin, J., Horvitz, H. R. \& Brenner, S. Nondisjunction mutants of the nematode Caenorhabditis elegans. Genetics 91, 67-94 (1979).

54. McNamee, L. M. \& Brodsky, M. H. p53-independent apoptosis limits DNA damage-induced aneuploidy. Genetics 182, 423-435 (2009).

55. Thonneau, P., Bujan, L., Multigner, L. \& Mieusset, R. Occupational heat exposure and male fertility: a review. Hum. Reprod. 13, 2122-2125 (1998).

56. Schoeller, E. L., Albanna, G., Frolova, A. I. \& Moley, K. H. Insulin rescues impaired spermatogenesis via the hypothalamic-pituitary-gonadal axis in Akita diabetic mice and restores male fertility. Diabetes 61, 1869-1878 (2012).

57. Coussons-Read, M. E. Effects of prenatal stress on pregnancy and human development: mechanisms and pathways. Obstet. Med. 6, 52-57 (2013).

58. Parker, V. J. \& Douglas, A. J. Stress in early pregnancy: maternal neuroendocrine-immune responses and effects. J. Reprod. Immunol. 85, 86-92 (2010).
59. Davis, E. P., Glynn, L. M., Waffarn, F. \& Sandman, C. A. Prenatal maternal stress programs infant stress regulation. J. Child Psychol. Psychiatry Allied Discip. 52, 119-129 (2011).

60. Weismann, A., Parker, W. N. \& Rōnníeldt, H. The Germ-plasm: A Theory of Heredity (C. Scribner's Sons, 1893).

61. Brenner, S. The genetics of Caenorhabditis elegans. Genetics 77, 71-94 (1974).

62. Stiernagle, T. Maintenance of C. elegans. WormBook 1-11 (2006).

63. Gumienny, T. L., Lambie, E., Hartwieg, E., Horvitz, H. R. \& Hengartner, M. O. Genetic control of programmed cell death in the Caenorhabditis elegans hermaphrodite germline. Development 126, 1011-1022 (1999).

64. Timmons, L. \& Fire, A. Specific interference by ingested dsRNA. Nature 395, 854 (1998).

65. Dupuy, D. et al. A first version of the Caenorhabditis elegans Promoterome. Genome Res. 14, 2169-2175 (2004).

66. Lee, M. H. \& Schedl, T. RNA in situ hybridization of dissected gonads. WormBook 1-7 (2006).

\section{Acknowledgements}

We thank M. Rieckher for support in planning the transgenics design and the CECAD imaging facility. Worm strains were provided by the National Bioresource Project (supported by The Ministry of Education, Culture, Sports, Science and Technology, Japan), the Caenorhabditis Genetics Center (funded by the NIH National Center for Research Resources, US), and the C. elegans Gene Knockout Project at the Oklahoma Medical Research Foundation (part of the International C. elegans Gene Knockout Consortium). N.S. received a fellowship from the Cologne Cardiovascular Research Center (CCRC), B.S. acknowledges funding from the Deutsche Forschungsgemeinschaft (SCHU 2494/3-1, SCHU 2494/7-1, SCHU 2494/10-1, SCHU 2494/11-1, CECAD EXC 2030 - 390661388, SFB 829, SFB 670, KFO 286, KFO 329, and GRK 2407), the Deutsche Krebshilfe (70112899), the H2020-MSCA-ITN-2018 (Healthage and ADDRESS ITNs), and the John Templeton Foundation Grant (61734).

\section{Author contributions}

N.S. and S.W. performed and analyzed the experiments and together with B.S. conceived and designed the study and wrote the manuscript.

\section{Funding}

Open Access funding enabled and organized by Projekt DEAL.

\section{Competing interests}

B.S. is co-founder of Agevio Therapeutics, Inc. All authors declare no other competing interests.

\section{Additional information}

Supplementary information The online version contains supplementary material available at https://doi.org/10.1038/s41467-022-28225-8.

Correspondence and requests for materials should be addressed to Björn Schumacher.

Peer review information Nature Communications thanks the anonymous reviewers for their contribution to the peer review of this work.

Reprints and permission information is available at http://www.nature.com/reprints

Publisher's note Springer Nature remains neutral with regard to jurisdictional claims in published maps and institutional affiliations.

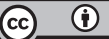

Open Access This article is licensed under a Creative Commons Attribution 4.0 International License, which permits use, sharing, adaptation, distribution and reproduction in any medium or format, as long as you give appropriate credit to the original author(s) and the source, provide a link to the Creative Commons license, and indicate if changes were made. The images or other third party material in this article are included in the article's Creative Commons license, unless indicated otherwise in a credit line to the material. If material is not included in the article's Creative Commons license and your intended use is not permitted by statutory regulation or exceeds the permitted use, you will need to obtain permission directly from the copyright holder. To view a copy of this license, visit http://creativecommons.org/ licenses/by/4.0/

(C) The Author(s) 2022, corrected publication 2023 